\title{
Cellules photo-électrochimiques à électrodes semiconductrices pour la conversion et le stockage de l'énergie solaire
}

\author{
G. Horowitz et A. Bourrasse \\ Centre d'Etudes Nucléaires de Saclay, Service d'Etudes Energétiques, BP 2, 91190 Gif sur Yvette, France
}

(Reşu le 31 août 1979, révisé le 17 décembre 1979, accepté le 18 décembre 1979)

\begin{abstract}
Résumé. - Discipline récente, la conversion photo-électrochimique (PEC) de l'énergie solaire a connu ces dernières années un important effort de recherche. Les cellules PEC se caractérisent par la présence d'électrodes semiconductrices. L'étude théorique de leur fonctionnement, et en particulier de la stabilité des électrodes semiconductrices, conduit à distinguer deux types de cellules : les cellules autorégénérées, fournissant de l'énergie électrique, et les cellules de stockage, permettant la génération d'espèces chimiques énergétiques. Les différentes réalisations de cellule solaire PEC, ainsi que les rendements obtenus, sont passés en revue.
\end{abstract}

\begin{abstract}
In the last years, an important research effort has developed on the photoelectrochemical (PEC) conversion of solar energy. PEC cells are characterized by the presence of semiconductor-electrodes. The theoretical study of their mechanisms, and specially the stability of semiconductor electrodes, leads to the distinction between two kinds of cells : Regenerative cells, which produce electrical energy, and storage cells, which can generate energetic chemical species. The PEC solar cells reported in the litterature, and the power efficiencies that have been obtained, are reviewed.
\end{abstract}

1. Introduction. - En 1972, Fujishima et Honda [1] faisaient paraître dans la revue Nature une lettre dans laquelle ils décrivaient une cellule électrochimique comprenant une anode constituée par un cristal semiconducteur de rutile $\mathrm{TiO}_{2}$. Cette cellule était capable de réaliser l'électrolyse de l'eau lorsque son électrode semiconductrice était exposée au rayonnement solaire. Elle montrait la possibilité de production d'hydrogène à partir de l'énergie solaire, et donc de conversion directe de cette énergie en énergie chimique stockable.

Par ailleurs, Gerischer [2] décrivait en 1975 une cellule solaire photo-électrochimique à électrode semiconductrice, tout à fait comparable à une cellule photovoltaïque, de type $p-n$ ou Schottky, à ceci près que la jonction solide-solide $\mathrm{y}$ était remplacée par une jonction solide-liquide (semiconducteur-électrolyte). Trois semiconducteurs différents ont été utilisés par Gerischer : GaP, CdS et CdSe, tous de type $n$. Malheureusement, les performances de ces cellules se dégradaient assez rapidement, car on observait une décomposition des électrodes semiconductrices lors de leur fonctionnement.

Depuis 1975 , le nombre des publications sur la conversion et le stockage photo-électrochimique (PEC) de l'énergie solaire a crû de façon spectaculaire.
Plusieurs congrès, soit dans le domaine de la photochimie [3], soit dans celui de la conversion photovoltaïque [4] lui ont réservé une place, et l'un d'eux [5] lui a été entièrement consacré. Divers articles de revue ont également été publiés sur ce sujet $[6,35]$. On peut classer les différentes cellules PEC étudiées en deux grands groupes. Dans le premier, se trouvent les cellules de photoélectrolyse de l'eau. Elles comportent une, ou même deux, électrodes semiconductrices, qui ne doivent pas subir de décomposition lors du fonctionnement de la cellule. Ce sont des cellules de stockage de l'énergie solaire. Le second groupe contient les cellules photovoltaïques à jonction liquide, telles que celles décrites par Gerischer, pour lesquelles le problème est de choisir judicieusement l'électrolyte, de façon à stabiliser l'électrode semiconductrice, c'est-à-dire d'empêcher sa décomposition lors du fonctionnement de la cellule.

Dans cet article, nous ferons le point des recherches entreprises sur ces deux types de cellule PEC. Dans un premier temps, nous décrirons les mécanismes qui interviennent au cours de la conversion PEC, ainsi que les moyens d'étude de ces mécanismes. Puis nous passerons en revue les cellules PEC de chaque groupe, et nous tenterons de dégager les tendances actuelles dans ce domaine. 
2. Principes de la conversion photo-électrochimique. -2.1 LA JONCTION SEMICONDUCTEUR-ÉLECTROLYTE. - Lorsqu'on met en contact un semiconducteur et un électrolyte, l'équilibre thermodynamique est atteint par l'égalisation des potentiels chimiques, qui se traduit par un échange électronique entre les deux milieux [7]. Dans le semiconducteur, cela conduit à la création d'une couche appauvrie en porteurs majoritaires, ou zone de charge d'espace, dont la largeur peut varier de $100 \AA$ pour un semiconducteur fortement dopé $\left(10^{19}\right.$ porteurs $\left.\mathrm{cm}^{-3}\right)$ à quelques microns s'il est faiblement dopé $\left(10^{16} \mathrm{~cm}^{-3}\right)$. Une couche similaire, constituée d'ions de charge opposée à celle de la zone de charge d'espace, existe du côté de l'électrolyte. Lorsque ce dernier a une concentration en ions supérieure à 0,1 mole (soit environ $10^{20}$ ions $\mathrm{cm}^{-3}$ ), ce qui sera toujours le cas dans une cellule PEC, pratiquement toute la charge ionique se trouve concentrée dans la couche de Helmholtz, c'est-à-dire dans un plan situé à quelques Å de l'interface avec le semiconducteur.

La figure 1 montre un diagramme énergétique de l'interface semiconducteur-électrolyte. Le potentiel chimique du semiconducteur, c'est-à-dire son niveau de Fermi $E_{\mathrm{F}, \mathrm{sc}}$ est défini par rapport à l'énergie de l'électron dans le vide. Dans l'électrolyte, $E_{\mathrm{F} \text {,redox }}$ correspond au potentiel redox des couples oxydoréducteurs présents dans la solution; il est défini par rapport à une électrode de référence qui est par convention l'électrode normale à hydrogène (NHE). (Dans la pratique, on utilise souvent l'électrode au calomel saturé (SCE).) Les deux échelles, celle du physicien des solides et celle de l'électrochimiste, peuvent être mises en coïncidence par la détermination de la différence de potentiel $\Delta V_{\mathrm{R}}$ entre le niveau du vide et le niveau redox de référence. Des valeurs allant de $-4,5 \mathrm{~V}$ [8] à $-4,73$ [9] ont été obtenues pour l'électrode normale à hydrogène.

On peut montrer que la chute de potentiel à travers

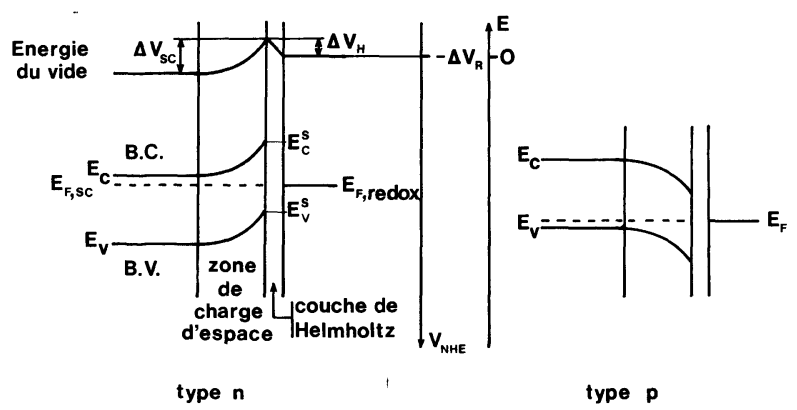

Fig. 1. - Schéma énergétique de l'interface semiconducteur-électrolyte pour un semiconducteur de type $n$ et p. B.C. : bande de conduction, B.V. : bande de valence. Les deux échelles d'énergie sont celle de l'électrochimie (à gauche) et celle de la physique des solides (à droite).

[Schematic energy diagram for the semiconductor-electrolyte interface, for $\mathrm{n}$-and p-type semiconductor. B.C. : conduction band, B.V. : valence band. The two energy scales are the electrochemist (left hand side) and the solid state physicist (right hand side).] la couche de Helmholtz ne dépend pratiquement que de la composition de l'électrolyte, et non de la charge d'espace du semiconducteur $[10,11]$. En conséquence, lorsqu'on fait varier le potentiel de l'électrode semiconductrice par l'application d'une tension extérieure, seule varie la chute de potentiel à travers sa zone de charge d'espace.

Les niveaux des bords des bandes $E_{\mathrm{c}}^{\mathrm{s}}$ et $E_{\mathrm{v}}^{\mathrm{s}}$ à la surface du semiconducteur restent fixes par rapport au niveau de Fermi de l'électrolyte, et donc aussi par rapport à l'électrode de référence. La détermination du potentiel de bande plate, $V_{\mathrm{fb}}$, c'est-à-dire du niveau de Fermi du semiconducteur lorsque la chute de potentiel à travers sa zone de charge d'espace s'annule, permet donc la détermination de $E_{\mathrm{c}}^{\mathrm{s}}$ et $E_{\mathrm{v}}^{\mathrm{s}}$.

\subsection{DÉTERMINATION DU POTENTIEL DE BANDES PLA-} TES. - La méthode classique de détermination de $V_{\mathrm{fb}}$ consiste à mesurer la capacité différentielle par unité de surface de l'interface semiconducteurélectrolyte en fonction du potentiel de l'électrode semiconductrice. Si l'on ne tient pas compte des états localisés pouvant exister à la surface du semiconducteur, la capacité de l'interface peut être représentée par le schéma équivalent de la figure $2 a$ [12]. $C_{\mathrm{H}}$ est la capacité par unité de surface de la couche de Helmholtz, $C_{\text {sc }}$ celle de la zone de charge d'espace, $Z_{\mathrm{f}}$ une impédance de fuite, qui peut être négligée à haute fréquence, et $R_{\mathrm{s}}$ la résistance série de l'électrolyte et du semiconducteur. Du fait de sa très faible épaisseur (quelques $\AA$ ), la couche de Helmholtz a une capacité très grande devant $C_{\mathrm{sc}}$. A haute fréquence, on est ramené au schéma de la figure $2 b$, où $C_{\mathrm{sc}}$ apparaît comme la capacité série de l'interface. Pour un semiconducteur dopé, on a la relation de MottSchottky [13] :

$$
C_{\mathrm{sc}}^{-2}=\left(1 / 2 \varepsilon \varepsilon_{0} e N\right)\left(V-V_{\mathrm{fb}}-k T / e\right)
$$

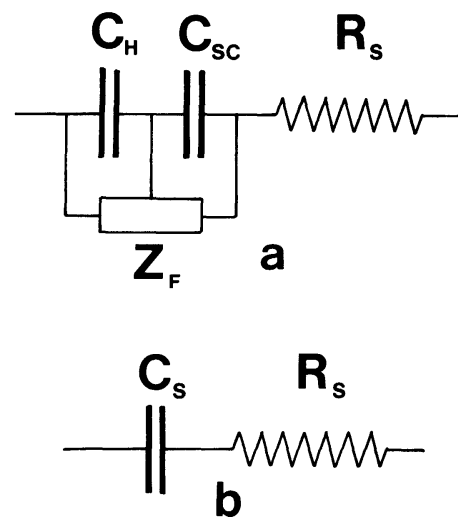

Fig. 2. - Schéma équivalent : a) de l'interface semiconducteurélectrolyte, $C_{\mathrm{H}}$ : capacité de la couche de Helmholtz, $C_{\mathrm{sc}}$ : capacité de la zone de charge d'espace, $Z_{\mathrm{f}}:$ impédance de fuite, $R_{\mathrm{s}}:$ résistance série; $b$ ) de l'interface à haute fréquence.

[Equivalent circuit : $a$ ) of the semiconductor electrolyte interface $C_{\mathrm{H}}$ : Helmholtz layer capacitance, $C_{\mathrm{sc}}$ : space-charge layer capacitance, $Z_{\mathrm{f}}$ : leakage impedance, $R_{\mathrm{s}}$ : series resistance; $b$ ) of the interface at high frequency.] 


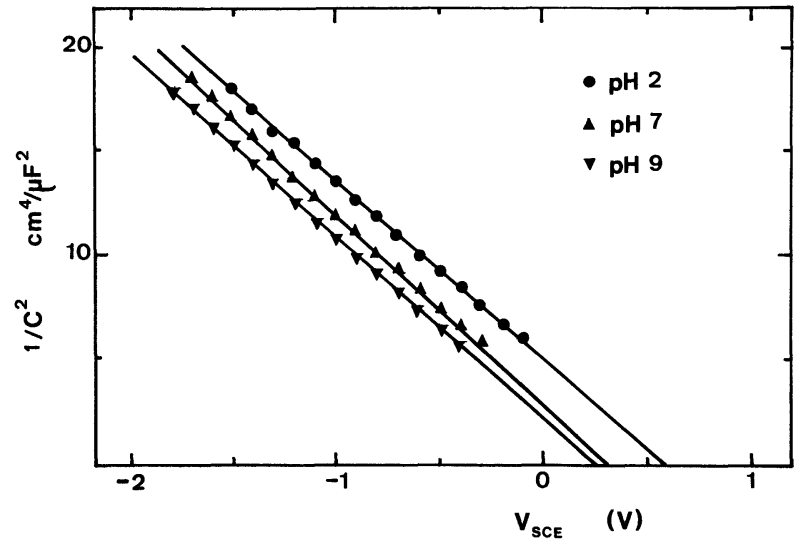

Fig. 3. - Variation de $1 / C_{\mathrm{sc}}^{2}$ en fonction du potentiel d'électrode par rapport à l'électrode SCE pour une électrode de GaP-p dans une solution électrolytique tamponnée, à trois valeurs différentes du $\mathrm{pH}$. Les droites de Mott-Schottky ont été obtenues par ajustement au sens des moindres carrés.

[Mott-Schottky plot of the capacitance of a p-type GaP electrode in a buffered electrolytic solution at three different $\mathrm{pH}$ levels, as a function of the electrode potential relative to SCE. The straight lines have been obtained by least square fit.]

où $N$ est la densité de porteurs majoritaires, $\varepsilon$ la constante diélectrique, $V$ le potentiel par rapport à une électrode de référence, et $V_{\mathrm{fb}}$ le potentiel de bande plate. La figure 3 donne les résultats de mesures que nous avons faites sur une électrode en $\mathrm{GaP}$ de type $\mathrm{p}$ [14] dans une solution tamponnée à différents $\mathrm{pH}$. $V_{\mathrm{fb}}$ s'obtient, à $k T / e$ près, par extrapolation de la droite à $1 / C^{2}=0$. La figure 4 montre la variation de $V_{\mathrm{fb}}$ avec le $\mathrm{pH}$. Cette variation est linéaire, et suit la loi de Nernst : $-0,059 \mathrm{~V}$ par degré $\mathrm{pH}$. Elle est due à la variation de la chute de potentiel $\Delta V_{H}$ à travers la couche de Helmholtz, qui selon Gerischer [7] s'explique par le caractère acide ou basique de la surface du semiconducteur. Celle-ci peut réagir avec

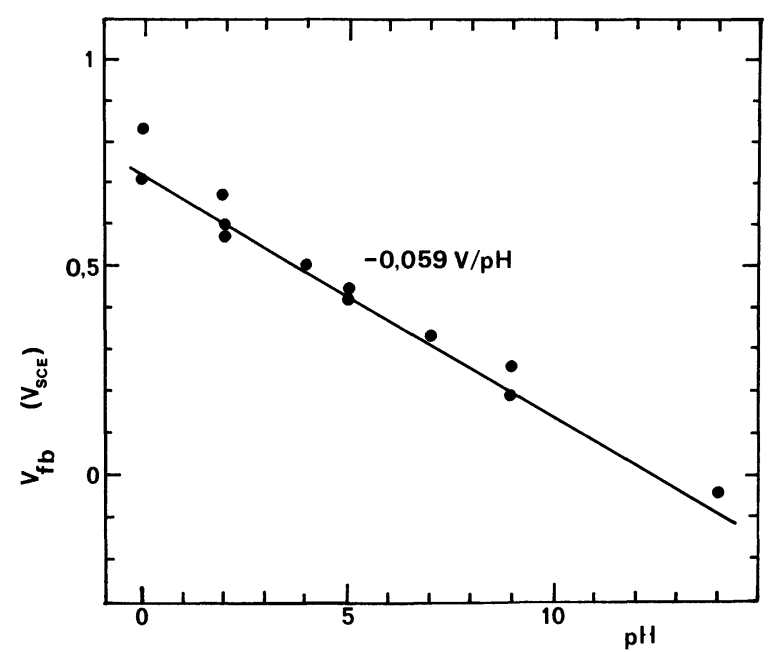

Fig. 4. - Variation du potentiel de bande plate d'une électrode en GaP-p en fonction du $\mathrm{pH}$ de l'électrolyte.

[Variation of the flat-band potential of a p-type $\mathrm{GaP}$ electrode as a function of the $\mathrm{pH}$ level.] l'eau en s'associant à un proton ou à un ion hydroxyle. Dans ce dernier cas, par exemple, on a la relation d'équilibre :

$$
\mathrm{A}-\mathrm{OH}+\mathrm{H}_{2} \mathrm{O} \rightleftarrows \mathrm{AO}^{-}+\mathrm{H}_{3} \mathrm{O}^{+}
$$

où $\mathrm{A}$ est un atome de la surface du semiconducteur. La réaction (2) correspond à un transfert de charges vers la couche de Helmholtz et donc à une variation de $\Delta V_{\mathrm{H}}$, qui est alors contrôlé par le $\mathrm{pH}$ selon la relation :

$$
\Delta V_{\mathbf{H}}=\mathrm{Cte}+(2,3 k T / e) \mathrm{pH}
$$

soit $\Delta V_{\mathrm{H}}=\mathrm{Cte}+0,059 \mathrm{pH}$ à température ambiante.

Le même genre de dépendance de $V_{\mathrm{fb}}$ a pu être observé lorsque la solution électrolytique contient un autre couple redox susceptible de s'associer à la surface du semiconducteur. Ainsi, dans le cas de CdS en présence du sulfure, $V_{\mathrm{fb}}$ varie avec la concentration en ions sulfures dans l'électrolyte [15].

Connaissant $V_{\mathrm{fb}}, E_{\mathrm{c}}^{\mathrm{s}}\left(E_{\mathrm{v}}^{\mathrm{s}}\right)$ est déterminé par la différence d'énergie entre le niveau de Fermi et le bas de la bande de conduction pour un semiconducteur de type $\mathrm{n}$ (le haut de la bande de valence pour un semiconducteur de type $p$ ). Cette différence est donnée, pour un semiconducteur non dégénéré, par [16] :

$$
\begin{aligned}
& E_{\mathrm{c}}-E_{\mathrm{F}}=k T \ln \left(N_{\mathrm{c}} / n_{0}\right) \quad(\underline{\text { type } \mathrm{n}}) \\
& E_{\mathrm{F}}-E_{\mathrm{v}}=k T \ln \left(N_{\mathrm{v}} / p_{0}\right) \quad(\underline{\text { type })})
\end{aligned}
$$

où $n_{0}\left(p_{0}\right)$ est la densité d'électrons (trous) libres et $N_{\mathrm{c}}\left(N_{\mathrm{v}}\right)$ la densité d'états en bas de la bande de conduction (haut de la bande de valence). La distance entre les bords de bande sera donnée par la largeur $E_{\mathrm{g}}$ de la bande interdite. La position de $E_{\mathrm{c}}^{\mathrm{s}}$ et $E_{\mathrm{v}}^{\mathrm{s}}$ de différents semiconducteurs à pH 7 est donnée par la figure 5 .

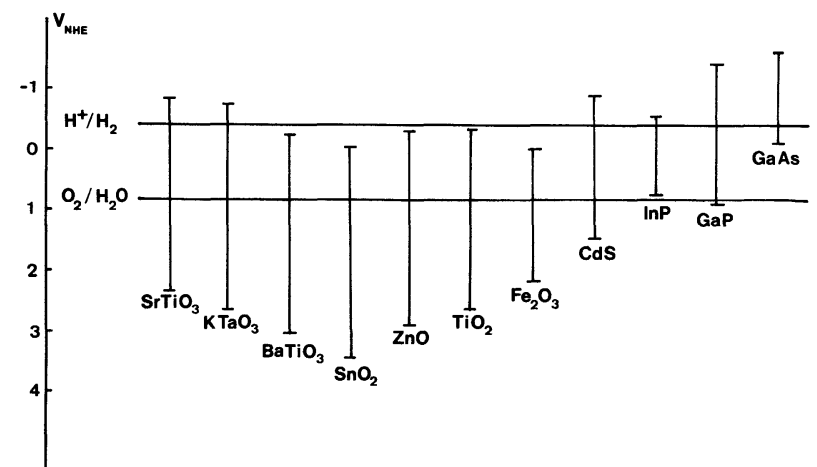

Fig. 5. - Position des bords de bandes à la surface de différents semiconducteurs en contact avec un électrolyte à $\mathrm{pH} 7$. Les positions ont été calculées à partir des mesures de potentiel de bande plate par la méthode de Mott-Schottky par Cardon et Gomes [17] pour $\mathrm{TiO}_{2}, \mathrm{GaP}, \mathrm{GaAs}, \mathrm{InP}, \mathrm{ZnO}$ et CdS, par Bolts et Wrighton [18] pour $\mathrm{SnO}_{2}, \mathrm{KTaO}_{3}$ et $\mathrm{SrTiO}_{3}$, par Kennedy et Freze [19] pour $\mathrm{BaTiO}_{3}$ et par Quinn et Nasby [20] pour $\mathrm{Fe}_{2} \mathrm{O}_{3}$.

[Band-edge position of several semiconductors at $\mathrm{pH}$ 7. The positions have been calculated from flat-band potential determinations obtained by capacitance measurements by Cardon and Gomes [17] for $\mathrm{TiO}_{2}, \mathrm{GaP}, \mathrm{GaAs}, \mathrm{InP}, \mathrm{ZnO}$ and $\mathrm{CdS}$, by Bolts and Wrighton [18] for $\mathrm{SnO}_{2}, \mathrm{KTaO}_{3}$ and $\mathrm{SrTiO}_{3}$, by Kennedy and Freze [19] for $\mathrm{BaTiO}_{3}$ and by Quinn and Nasby [20] for $\mathrm{Fe}_{2} \mathrm{O}_{3}$.] 
Nous avons établi cette figure à partir de valeurs du potentiel de bande plate obtenues par mesure de capacité différentielle [17-20]. Remarquons que les niveaux représentés sont ceux existant à la surface du semiconducteur, et qu'on ne peut pas les comparer au niveau du vide. Il faudrait pour cela connaître $\Delta V_{\mathrm{H}}$, dont la valeur, pour un électrolyte donné, peut varier d'un semiconducteur à l'autre [21].

D'autres méthodes de détermination de $V_{\mathrm{fb}}$ sont possibles, faisant appel à des mesures de photocourant [22] ou de photopotentiel [23]. Bien que moins précises, elles peuvent se révéler intéressantes lorsque les mesures de capacité différentielle sont délicates, par exemple dans le cas d'électrodes polycristallines.

2.3 ECHANGeS ÉLECTRONIQUES À L'INTERFACE SEMICONDUCTEUR-ÉLECTROLYTE. - L'échange électronique entre une électrode et l'électrolyte est un phénomène plus rapide que le réarrangement de la gaine d'atomes du solvant entourant les ions présents dans l'électrolyte. Suivant le principe de Franck-Condon, cet échange aura donc lieu sans changement de la configuration chimique des ions. Il doit d'autre part être isoénergétique, c'est-à-dire qu'il ne peut intervenir qu'entre états de même énergie, à $k T$ près [7].

La densité d'états vides et occupés dans l'électrolyte peut être décrite par un modèle simple dû à Gurney [24] (Fig. 6). Les niveaux vides (accepteurs) correspondent à l'espèce oxydée du couple redox, les niveaux pleins (donneurs) à l'espèce réduite. La différence d'énergie entre les niveaux vides et pleins les plus probables, correspond à la différence d'énergie de solvatation des espèces oxydée et réduite. Les densités d'états sont données par [25, 26] :

$$
D_{\text {ox }}(E)=n_{\mathrm{ox}}(4 \pi \lambda k T)^{-1 / 2} \exp \left[-\left(E-E_{\mathrm{ox}}^{0}\right)^{2} / 4 \lambda k T\right]
$$

$D_{\text {red }}(E)=n_{\text {red }}(4 \pi \lambda k T)^{-1 / 2} \exp \left[-\left(E-E_{\text {red }}^{0}\right)^{2} / 4 \lambda k T\right]$

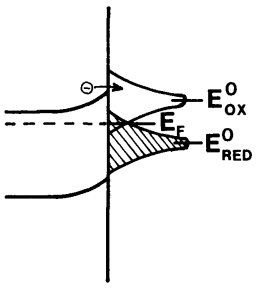

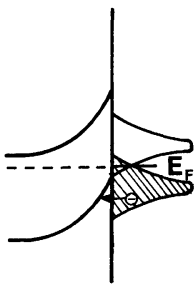

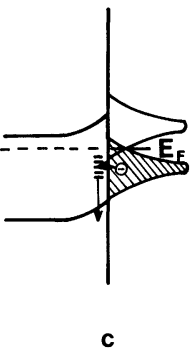

Fig. 6. - Densité d'états vides et occupés d'un électrolyte, et processus d'échange électronique à l'interface, avec un semiconducteur : $a$ ) faible courbure des bandes (échange avec les porteurs majoritaires) ; $b$ ) forte courbure des bandes ; $c$ ) échange par l'intermédiaire d'états de surface.

[Density of empty and occupied states in an electrolyte, and electronic transfer process at a semiconductor surface $: a$ ) weak band bending; $b$ ) strong band bending; $c$ ) transfer via surface states.] où $E_{\mathrm{ox}}^{0}$ et $E_{\mathrm{red}}^{0}$ sont les énergies des états les plus probables, $n_{\text {ox }}$ et $n_{\text {red }}$ la concentration des espèces oxydée et réduite. $\lambda$ représente l'énergie de réarrangement de la gaine solvatée entre le niveau d'équilibre $E_{\mathrm{F}, \text { redox }}$ et l'état le plus probable.

La figure $6 a$ montre la position des bandes d'énergies d'un semiconducteur de type $\mathrm{n}$ par rapport aux densités d'états de l'électrolyte. Les échanges ne peuvent se faire aisément qu'avec la bande de conduction (porteurs majoritaires). Le transfert de porteurs minoritaires aura lieu dans le cas d'une forte courbure de bande (Fig. 6b) ou par l'intermédiaire d'états localisés à la surface du semiconducteur (Fig. 6c).

\subsection{LA JONCTION SEMICONDUCTEUR ÉLECTROLYTE} SOUS ILLUMINATION. - Lorsqu'on éclaire la surface du semiconducteur avec une lumière d'énergie supérieure à la largeur de la bande interdite, les photons sont absorbés en créant une paire électron-trou qui se sépare sous l'effet du champ électrique existant dans la zone de charge d'espace. Cela se traduit par l'apparition d'un photopotentiel $\Delta V_{\text {ph }}$ qui a pour effet de diminuer la courbure des bandes à la surface du semiconducteur.

Si l'électrode semiconductrice est reliée à une contre-électrode par un circuit extérieur, un photocourant pourra circuler dans ce circuit à la condition que les porteurs minoritaires arrivant à la surface du semiconducteur passent en solution par l'intermédiaire d'une réaction électrochimique avec les ions présents dans l'électrolyte.

La forme générale de la caractéristique intensitépotentiel d'une jonction semiconducteur-électrolyte est donnée par la figure 7. Dans l'obscurité, la jonction agit comme une diode. Le courant dû aux porteurs majoritaires (courant cathodique pour un semiconducteur de type n, anodique pour le type p), est analogue à celui d'une électrode métallique, tandis que le courant des porteurs minoritaires est quasi-

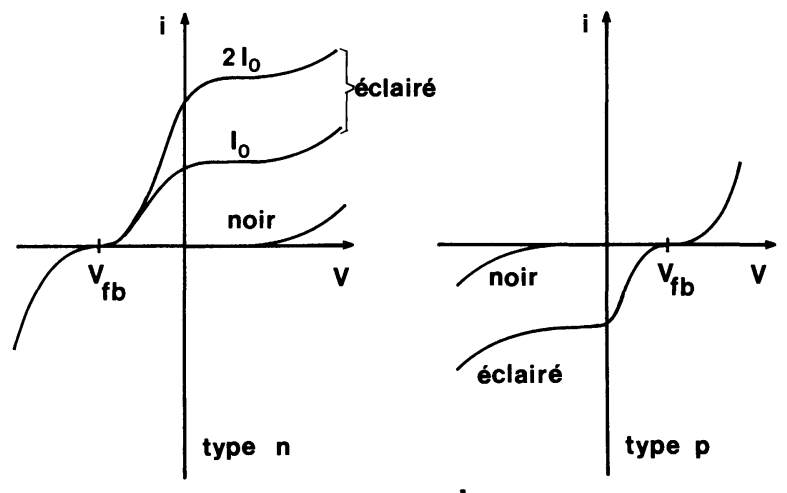

a

b

Fig. 7. - Caractéristiques intensité-potentiel d'une jonction semiconducteur-électrolyte, dans le noir et sous éclairement : $a$ ) type n, b) type $\mathrm{p}$.

[Current-voltage characteristic of a semiconductor-electrolyte junction in the dark and under illumination : $a$ ) n-type, $b$ ) p-type.] 
ment nul. Remarquons, toutefois, qu'à forte polarisation inverse (anodique pour le type $\mathrm{n}$, cathodique pour le type $p$ ), il y a apparition d'un courant qui peut être dû soit à l'effet tunnel des porteurs majoritaires, soit à un phénomène d'avalanche.

Lorsque l'électrode est éclairée à une énergie plus grande que $E_{\mathrm{g}}$, on observe un courant en polarisation inverse, qui commence à un potentiel voisin du potentiel de bande plate, et qui atteint ensuite une valeur de saturation. Un modèle simple, issu de la théorie de Gärtner [27], permet d'expliquer l'allure de ces caractéristiques sous illumination.

Dans ce modèle, la densité de courant $j$ est donnée par [22] :

$$
j=e \varphi_{0}\left[1-\exp (-\alpha W) /\left(1+\alpha L_{\mathrm{p}}\right)\right]
$$

où $\varphi_{0}$ est le flux de photons incidents, $\alpha$ le coefficient d'absorption optique,

$$
W=\left(2 \varepsilon \varepsilon_{0} / e N\right)^{1 / 2}\left(V-V_{\mathrm{fb}}+k T / e\right)^{1 / 2}
$$

la largeur de la zone de charge d'espace, et $L_{\mathrm{p}}$ la longueur de diffusion des porteurs minoritaires.

Dans une approximation supplémentaire, on peut négliger $\alpha \mathrm{L}_{\mathrm{P}}$ devant 1 , ce qui revient à dire que la longueur de diffusion des porteurs minoritaires est petite devant la longueur d'absorption des photons. Ceci n'est pas vrai pour des semiconducteurs comme $\mathrm{Si}$ et $\mathrm{Ge}$, mais semble vérifié dans le cas des composés utilisés en photo-électrochimie. On a alors simplement :

$j=e \varphi_{0}\left[1-\exp \left[-\alpha\left(2 \varepsilon \varepsilon_{0} k T / e N\right)^{1 / 2}\left(V-V_{\mathrm{fb}}\right)^{1 / 2}\right]\right]$.

La relation (7) décrit assez bien les caractéristiques sous illumination de la figure 7 . Le photocourant démarre à $V=V_{\mathrm{fb}}$, c'est-à-dire lorsqu'apparaît une courbure des bandes susceptible d'entraîner les porteurs minoritaires vers la surface du semiconducteur. Un courant de saturation est atteint lorsque $\alpha W>1$, c'est-à-dire lorsque tous les photoporteurs sont créés dans la zone de charge d'espace. Le rendement quantique $\eta_{\mathrm{q}}=j / e \varphi_{0}$, rapport du nombre de porteurs émis au nombre de photons incidents, et alors égal à 1 .

En utilisant le même modèle, mais avec d'autres conditions aux limites, qui tiennent compte en particulier des phénomènes de recombinaison des porteurs sur les états de surface du semiconducteur, Wilson [28] obtient un meilleur accord avec les caractéristiques mesurées. Cet accord est réalisé par l'ajustement de paramètres contrôlant le taux de recombinaison des porteurs en surface. Plusieurs approximations limitent l'approche du problème par la théorie de Gärtner. En particulier, elle ne tient compte ni du photopotentiel, qui modifie la courbure des bandes, ni de la recombinaison des porteurs à l'intérieur de la zone de charge d'espace.

D'autres approches théoriques sont possibles. Laser et Bard [29] ont effectué une simulation sur ordina- teur, consistant à résoudre numériquement l'équation de diffusion des porteurs minoritaires. Signalons, enfin, l'analyse très détaillée faite par Reiss [30], qui prend en compte aussi bien les phénomènes de recombinaison des porteurs que des mécanismes d'échange électronique à l'interface semiconducteur électrolyte. Cette analyse aboutit à des équations extrêmement complexes, dans lesquelles il est difficile de distinguer la part revenant à chacun des processus mis en jeu. Cela semble être, malheureusement, inévitable lorsque l'on veut inclure trop de facteurs différents dans une même analyse.

\subsection{STABILITÉ Des ÉLECTRODES SEMICONDUCTRICES} SOUS ÉCLAIREMENT. - Les cellules solaires PEC n'utilisent, bien entendu, pas comme électrolyte des solutions susceptibles d'attaquer chimiquement l'électrode semiconductrice. C'est seulement lorsqu'il y a passage d'un courant que cette dernière peut se décomposer électrochimiquement. Ce passage est, en effet, conditionné par la possibilité d'échange électronique à la surface du semiconducteur, soit avec des ions de la solution, soit avec des atomes du semiconducteur lui-même. C'est dans ce dernier cas, qu'il y a photodécomposition de l'électrode semiconductrice sous éclairement.

Deux articles parus simultanément, l'un dû à Gerischer [31], l'autre à Bard et Wrighton [32], ont donné des analyses assez voisines de la thermodynamique de la stabilité des électrodes semiconductrices. L'analyse de Gerischer a été reprise et développée par la suite [33]. Nous l'exposerons ici brièvement. La réaction de décomposition d'un semiconducteur peut intervenir comme réaction anodique (par les trous) ou cathodique (par les électrons).

Dans les deux cas, on peut la décrire comme un phénomène d'oxydoréduction, et lui associer un potentiel redox : $E_{\mathrm{dec}}^{\mathrm{p}}$ pour le processus anodique, $E_{\text {dec }}^{\mathrm{n}}$ pour la décomposition cathodique. La stabilité de la photoélectrode dépend de la position de ces niveaux par rapport aux bords des bandes. La figure 8 indique les quatre configurations possibles. L'élec-

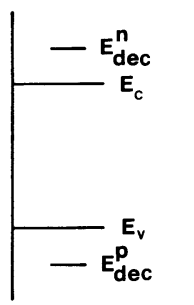

a

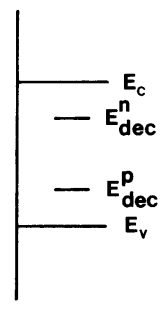

b

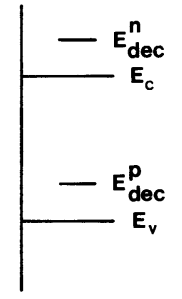

C

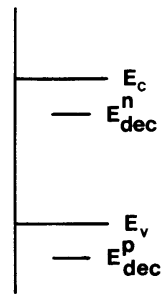

d
Fig. 8. - Critères thermodynamiques de stabilité des semiconducteurs en contact avec un électrolyte : $a$ ) stable; $b$ ) instable; c) stable en polarisation cathodique; $d$ ) stable en polarisation anodique.

[Thermodynamics of semiconductor photodecomposition : a) stable ; b) unstable; $c$ ) cathodically stable; $d$ ) anodically stable.] 
trode est stable anodiquement si $E_{\text {dec }}^{\text {p }}<E_{\mathrm{v}}$ et cathodiquement si $E_{\mathrm{dec}}^{\mathrm{n}}>E_{\mathrm{c}}$. Lorsque les niveaux de décomposition sont situés dans la bande interdite (instabilité : cas b, cet d), la décomposition se produit lorsque le niveau de Fermi est inférieur à $E_{\mathrm{dec}}^{\mathrm{p}}$ (décomposition anodique) ou si $E_{\mathrm{F}}>E_{\mathrm{dec}}^{\mathrm{n}}$ (décomposition cathodique). Lorsque le semiconducteur est éclairé, il faut selon Gerischer utiliser le pseudo-potentiel de Fermi $E_{\mathrm{F}}^{*}$ défini par :

$$
E_{\mathrm{F}}^{*}(\mathrm{n})=E_{\mathrm{c}}+k T \ln \left(n^{*} / N_{\mathrm{c}}\right)
$$

pour les électrons et

$$
E_{\mathrm{F}}^{*}(\mathrm{p})=E_{\mathrm{v}}-k T \ln \left(p^{*} / N_{\mathrm{v}}\right)
$$

pour les trous.

Ces relations ne sont autres que les relations (4) où les concentrations à l'équilibre sont remplacées par leur valeur $n^{*}\left(p^{*}\right)$ sous illumination. Le pseudopotentiel ne diffère du niveau de Fermi à l'équilibre que pour les porteurs minoritaires.

Cette approche qui repose sur une situation d'équilibre local, a été controversée par Williams et Nozik $[34,35]$ qui penchent plutôt pour un mécanisme d'échange fortement irréversible. Leur modèle assimile l'interface à une hétérojonction où l'électrolyte est traité comme un semiconducteur à large bande interdite [36]. L'injection des porteurs minoritaires vers l'électrolyte intervient par effet tunnel à un taux grand devant le taux de recombinaison des paires électron-trou.

Nozik fait en particulier remarquer que le modèle de Gerischer prévoit un seuil de photocourant dépendant de l'éclairement par l'intermédiaire des relations (8), puisque la densité de porteurs minoritaires varie avec l'intensité de l'illumination. Or, cette dépendance n'a pas été observée expérimentalement.

La réaction de décomposition d'un semiconducteur instable peut ne pas avoir lieu si est présent, dans

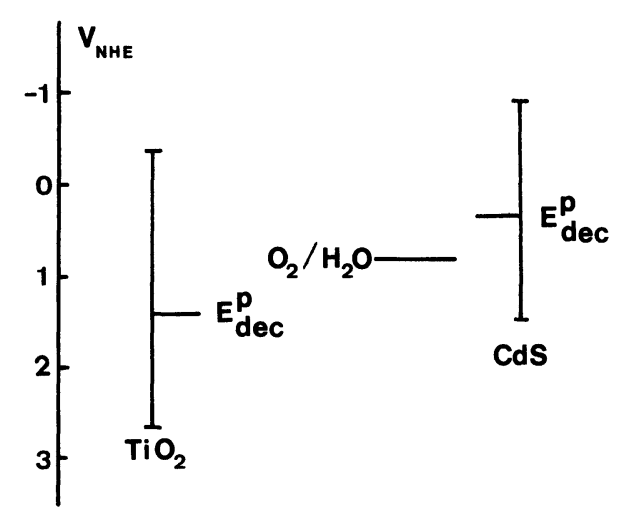

Fig. 9. - Schéma thermodynamique indiquant la stabilité de $\mathrm{TiO}_{2}$ et l'instabilité de CdS en polarisation anodique dans une solution aqueuse.

[Thermodynamic diagram showing that $\mathrm{TiO}_{2}$ is stable and $\mathrm{CdS}$ unstable in anodic polarisation under illumination in a water electrolyte.] l'électrolyte, un couple redox dont le potentiel est plus favorable à l'échange électronique que le potentiel de décomposition du semiconducteur. Ceci est illustré par la figure 9 : en polarisation anodique, $\mathrm{CdS}$ et $\mathrm{TiO}_{2}$ sont tous deux instables. Cependant, la présence du couple $\mathrm{O}_{2} / \mathrm{H}_{2} \mathrm{O}$ à un niveau plus cathodique que $E_{\text {dec }}^{\mathrm{p}}$ de $\mathrm{TiO}_{2}$ empêche la décomposition anodique de $\mathrm{TiO}_{2}$. Par contre, la décomposition de $\mathrm{CdS}$ aura lieu car dans ce cas, $E_{\text {dec }}^{\text {pest }}$ plus cathodique que le niveau $\mathrm{O}_{2} / \mathrm{H}_{2} \mathrm{O}$.

En réalité, les deux réactions - photo-décomposition et oxydoréduction du couple redox - ont lieu simultanément [37] et c'est la cinétique des échanges qui dictera la stabilité de l'électrode semiconductrice. Il faudra tenir compte à la fois de l'aspect thermodynamique et de l'aspect cinétique des réactions dans le choix d'un couple redox susceptible de stabiliser une électrode instable.

3. Les différents types de cellule solaires PEC. L'analyse de la stabilité des électrodes semiconductrices montre que deux types de cellules solaires PEC peuvent être envisagés. Les cellules du premier type utilisent un semiconducteur choisi pour sa bonne absorption optique aux longueurs d'onde correspondant au spectre solaire. Ce semiconducteur est généralement instable, et le problème consiste à trouver un couple redox susceptible de s'oxyder (ou de se réduire s'il s'agit d'un semiconducteur de type $p$ ) plus vite que le semiconducteur. A la contre-électrode, se produira alors la réaction inverse, c'est-à-dire qu'il y aura régénération de l'espèce oxydée (ou réduite). Le bilan chimique de cette cellule est donc nul, et il y a simplement production d'un courant électrique, comme dans le cas d'une cellule photovoltaïque.

Les cellules du deuxième type sont appelées cellules de stockage. Elles comportent un électrolyte contenant deux couples redox différents. Il y a oxydation du premier couple à l'anode et réduction du second à la cathode - l'une ou l'autre de ces électrodes (ou même les deux) étant semiconductrice et donc production d'espèces chimiques pouvant être stockées. Il n'est plus question dans ce cas de stabiliser une électrode instable. L'exemple le plus connu, et le plus étudié, est la cellule de photoélectrolyse décrite par Fusishima et Honda [1].

3.1 Cellules AUTORÉGÉNÉRÉES. - Le premier exemple de cellule PEC stabilisée par adjonction d'un couple redox adéquat à l'électrolyte, a été décrit paı Ellis, Kaiser et Wrighton [38].

Cette cellule est composée d'une anode en $\mathrm{CdS}$, et d'un électrolyte contenant du sulfure de sodium $\mathrm{Na}_{2} \mathrm{~S}$ et des polysulfures obtenus par dissolution de soufre. Le rendement de conversion (rapport de l'énergie électrique fournie à l'énergie lumineuse reçue par la cellule), mesurée en lumière monochromatique, est de $5 \%$ à $502 \mathrm{~nm}$. Avec une anode en CdSe dans le même électrolyte, le rendement de conversion atteint $9 \%$ à $633 \mathrm{~nm}$. 
Tableau I. - Cellules autorégénérées.

\begin{tabular}{|c|c|c|c|c|}
\hline Semiconducteur & $\begin{array}{c}\text { Couple Redox } \\
\text { stabilisateur } \\
\text { _ }\end{array}$ & Préparation de l'électrode & Rendement de conversion & Réf. \\
\hline \multirow[t]{10}{*}{$\mathrm{CdS}$} & $\mathrm{S}^{2-} / \mathrm{S}_{n}^{2-}$ & Monocristal & 5 à $6,8 \%$ à 502 nm & {$[38,39]$} \\
\hline & - & Monocristal & $1,3 \%$ au soleil & [40] \\
\hline & - & Electrode frittée & - & [41] \\
\hline & - & Couche mince anodique & - & {$[42,43]$} \\
\hline & - & $\begin{array}{l}\text { Couche mince } \\
\text { pulvérisation chimique }\end{array}$ & $0,5 \%$ à $480 \mathrm{~nm}$ & {$[44,45]$} \\
\hline & - & Id. - dopée au $\mathrm{Li}$ & $4 \%$ à $480 \mathrm{~nm}$ & [46] \\
\hline & - & $\begin{array}{l}\text { Couche mince } \\
\text { évaporation sous vide }\end{array}$ & - & [45] \\
\hline & $\mathrm{S}_{2} \mathrm{O}_{3}^{2} / \mathrm{S}_{4} \mathrm{O}_{6}^{2-}$ & Monocristal & - & {$[47]$} \\
\hline & $\mathrm{Se}^{2-} / \mathrm{Se}_{n}^{2-}$ & Monocristal & $3,4 \%$ à $502 \mathrm{~nm}$ & [48] \\
\hline & $\mathrm{Te}^{2-} / \mathrm{Te}_{n}^{2-}$ & Monocristal & $1,4 \%$ à $488 \mathrm{~nm}$ & [48] \\
\hline $\mathrm{CdS}-\mathrm{ZnSe}$ & $\mathrm{S}^{2-} / \mathrm{S}_{n}^{2-}$ & $\begin{array}{l}\text { Couche mince } \\
\text { évaporation sous vide }\end{array}$ & - & [49] \\
\hline$x \mathrm{CdS}-(1-x) \mathrm{CdSe}$ & $\mathrm{S}^{2-} / \mathrm{S}_{n}^{2-}$ & Electrode frittée & $9 \%$ à $577 \mathrm{~nm}(x=0,9)$ & {$[50]$} \\
\hline \multirow[t]{6}{*}{$\mathrm{CdSe}$} & $\mathrm{S}^{2-} / \mathrm{S}_{n}^{2-}$ & Monocristal & $9 \%$ à $633 \mathrm{~nm}$ & [38] \\
\hline & - & Monocristal & $8,1 \%$ au soleil & {$[40]$} \\
\hline & - & Electrode frittée & $5,1 \%$ au soleil & {$[51]$} \\
\hline & - & Couche mince anodique & $1 \%$ au soleil & {$[52]$} \\
\hline & $\mathrm{Se}^{2-} / \mathrm{Se}_{n}^{2-}$ & Monocristal & $2,1 \%$ à $633 \mathrm{~nm}$ & [48] \\
\hline & $\mathrm{Te}^{2-} / \mathrm{Te}_{n}^{2-}$ & Monocristal & $1,5 \%$ à $633 \mathrm{~nm}$ & [48] \\
\hline \multirow[t]{4}{*}{$\mathrm{CdTe}$} & $\mathrm{Se}^{2-} / \mathrm{Se}_{n}^{2-}$ & Monocristal & $9,2 \%$ à $633 \mathrm{~nm}$ & [48] \\
\hline & - & Monocristal & $8,4 \%$ au soleil & {$[53]$} \\
\hline & $\mathrm{Te}^{2-} / \mathrm{Te}_{n}^{2-}$ & Monocristal & $10-10,7 \%$ à $633 \mathrm{~nm}$ & {$[48,54]$} \\
\hline & - & $\begin{array}{l}\text { Couche mince } \\
\text { dépôt électrolytique }\end{array}$ & 2 à $4 \%$ lampe Xe & {$[55]$} \\
\hline GaP & $\mathrm{Se}^{2-} / \mathrm{Se}_{n}^{2-}$ & Monocristal & $2 \%$ à $454 \mathrm{~nm}$ & {$[56]$} \\
\hline \multirow[t]{3}{*}{ GaAs } & $\mathrm{Te}^{2-} / \mathrm{Te}_{n}^{2-}$ & Monocristal & $3,8 \%$ à $633 \mathrm{~nm}$ & [56] \\
\hline & $\mathrm{Se}^{2-} / \mathrm{Se}_{n}^{2-}$ & Monocristal & $8,8 \%$ au soleil & {$[57]$} \\
\hline & - & $\begin{array}{l}\text { Monocristal plongé dans } \\
\mathrm{Ru} \text { (III) }\end{array}$ & $12 \%$ au soleil & {$[58]$} \\
\hline InP & $\mathrm{Te}^{2-} / \mathrm{Te}_{n}^{2-}$ & Monocristal & $1 \%$ à $633 \mathrm{~nm}$ & [59] \\
\hline $\mathrm{Bi}_{2} \mathrm{~S}_{3}$ & $\mathrm{~S}^{2-} / \mathrm{S}_{n}^{2-}$ & Couche mince anodique & - & [42] \\
\hline $\mathrm{CuInS}_{2}$ & $\mathrm{~S}^{2-} / \mathrm{S}_{n}^{2-}$ & $\begin{array}{l}\text { Monocristal } \\
\text { Electrode frittée }\end{array}$ & $3,5-4,3 \%$ lampe $W\}$ & {$[60]$} \\
\hline GaP type $p$ & $\mathrm{Eu}^{2+} / \mathrm{Eu}^{3+}$ & Monocristal & - & [37] \\
\hline $\mathrm{MoSe}_{2}$ & $\mathrm{I}^{-} / \mathrm{I}_{2}$ & Monocristal & $3,5 \%$ au soleil & [78] \\
\hline
\end{tabular}

De nombreuses publications sur ce type de cellule ont vu le jour depuis. Nous indiquons, dans le tableau I, les principaux semiconducteurs qui ont été employés, ainsi que les couples redox stabilisateurs et le mode de fabrication de l'électrode. Les rendements les plus élevés ont été mesurés sur des électrodes monocristallines, mais on peut remarquer que les performances réalisées avec des électrodes polycristallines sont assez voisines. Elles dépendent, en outre, de la préparation de ces électrodes, et sont donc susceptibles d'être améliorées.

3.1.1 Mode de préparation des électrodes semiconductrices. - Les électrodes polycristallines sont obtenues par frittage $[41,50,51,60]$ ou par diverses techniques de préparation de couches minces :

- Evaporation sous vide [45, 49] sur un substrat de verre recouvert d'oxyde d'étain $\mathrm{SnO}_{2}$ conducteur, servant de contact électrique à l'électrode semiconductrice.

- Pulvérisation chimique [44-46] (méthode dite du spray [61]), également sur substrat en verre recouvert de $\mathrm{SnO}_{2}$. Ce substrat étant transparent, il est possible d'éclairer l'électrode à travers le substrat plutôt qu'à travers l'électrolyte [62, 63].

- Dépôt anodique d'un des composants sur une électrode métallique constituée par l'autre composant du semiconducteur. Des couches minces de CdS [42, 43], CdSe [52] et $\mathrm{Bi}_{2} \mathrm{~S}_{3}$ [42] ont été ainsi réalisées.

- Dépôt électrolytique des deux composants sur un substrat métallique (titane) [55].

3.1.2 Choix du couple redox stabilisateur. - La stabilisation des électrodes est obtenue principalement 
par des chalcogénures, dont le potentiel redox est fortement cathodique :

$$
\begin{array}{cc}
\mathrm{S}^{2-} / \mathrm{S}_{n}^{2-}\left(-0,7 V_{\mathrm{SCE}}\right), \mathrm{Se}^{2-} / \mathrm{Se}_{n}^{2-}\left(-0,9 V_{\mathrm{SCE}}\right) \\
\text { et } & \mathrm{Te}^{2-} / \mathrm{Te}_{n}^{2-}\left(-1,1 V_{\mathrm{SCE}}\right)
\end{array}
$$

La position respective de ces niveaux et des niveaux de décomposition anodique des semiconducteurs donne un critère thermodynamique de stabilité. On explique ainsi que $\mathrm{GaP}$ soit instable dans $\mathrm{S}^{2-} / \mathrm{S}_{n}^{2-}$ et stable dans $\mathrm{Se}^{2-} / \mathrm{Se}_{n}^{2-}$ et dans $\mathrm{Te}^{2-} / \mathrm{Te}_{n}^{2-}$ [62]. La cinétique des réactions peut cependant intervenir dans le phénomène de stabilisation, et ce d'autant plus que les niveaux redox et de décomposition sont voisins.

La mesure des vitesses de réaction intervenant à la surface du semiconducteur est possible par la méthode de l'électrode tournante [65], qui permet de déterminer le rapport entre le courant d'oxydation du couple redox et le courant de décomposition de l'électrode. Inoue et al. [47] ont ainsi déterminé le taux de stabilisation du CdS par différents couples redox. Ils ont montré que $\mathrm{CdS}$ est stabilisé par $\mathrm{SO}_{3}^{2-} / \mathrm{SO}_{4}^{2-}, \mathrm{S}^{2-} / \mathrm{S}_{n}^{2-}$ et $\mathrm{S}_{2} \mathrm{O}_{3}^{2-} / \mathrm{S}_{4} \mathrm{O}_{6}^{2-}$, ce dernier étant le plus intéressant, car il possède le niveau redox le plus anodique. Par la même méthode, Memming [37] a étudié les semiconducteurs de type $\mathrm{p}$. Ceux-ci sont généralement stables en polarisation cathodique, et peuvent être utilisés dans les cellules de stockage. Cependant, la forte courbure de bande qu'ils présentent en contact avec certains couples redox en font aussi des candidats pour une cellule autorégénérée. C'est ainsi qu'avec une cellule GaP$\mathrm{p} /\left(\mathrm{Eu}^{2+} / \mathrm{Eu}^{3+}\right) / \mathrm{Au}$, une tension en circuit ouvert de $1,07 \mathrm{~V}$ a été mesurée. C'est une des plus fortes valeurs obtenues avec une cellule PEC.

La stabilisation de CdSe par $\mathrm{S}^{2-} / \mathrm{S}_{n}^{2-}$, observée par plusieurs équipes $[38,40,51,52]$ a été mise en doute par Gerischer et Gobrecht [66]. L'étude de la variation à long terme de la réponse spectrale d'une telle cellule leur fait penser qu'il y a échange d'atomes de soufre et de sélénium entre l'électrolyte et la surface du semiconducteur, et formation sur celle-ci d'une couche de $\mathrm{CdS}$, donnant lieu à une dégradation des performances de la cellule.

Cette couche est fortement désordonnée du fait du désaccord important entre les paramètres cristallins des deux chalcogénures. La substitution de Se en $\mathrm{S}$ a été également observée par Heller et al. [67], par spectroscopie Auger, et par Cahen et al. [68], par spectroscopie photo-électronique (XPS). L'épaisseur de la couche désordonnée de CdS est estimée par ces derniers à quelques dizaines d'Å. Ils baptisent la structure ainsi obtenue EIS (électrolyte, isolant, semiconducteur) par analogie avec MIS (métal, isolant, semiconducteur), et pensent que le rendement pourrait en être amélioré, de la même manière que le rendement des cellules solaires MIS-tunnel est meilleur que celui des cellules de type Schottky [69].
Plus récemment, Noufi et al. [70] ont observé la substitution inverse : formation d'une couche de CdSe à la surface d'une électrode de CdS stabilisée par $\mathrm{Se}^{2-} / \mathrm{Se}_{n}^{2-}$.

3.1.3 Allure des caractéristiques intensité-potentiel. - Le meilleur rendement de conversion solaire a été obtenu avec GaAs monocristallin stabilisé par $\mathrm{Se}^{2-} / \mathrm{Se}_{n}^{2-}[57,58]$. Nous avons fait des mesures de caractéristiques intensité-potentiel sur une cellule de ce type. La caractéristique obtenue sous illumination par une lampe à vapeur de Xe avec une anode en GaAs polycristallin non dopé (densité de porteurs de l'ordre de $10^{16} \mathrm{~cm}^{3}$ ) dans $\mathrm{Se}^{2-} / \mathrm{Se}_{n}^{2-}$ sont reproduites sur la figure 10. La caractéristique de la contre-électrode en platine a été également mesurée. Lorsque les deux électrodes sont reliées par un circuit extérieur, la cellule fonctionne entre les points situés aux extrémités de droites de charge, de telle sorte que les courants anodique et cathodique soient égaux. En court-circuit, le fonctionnement correspond à la droite verticale. $\mathrm{Si}$ une résistance de charge est placée dans le circuit extérieur, la pente de la droite de charge correspond à la valeur de cette résistance.

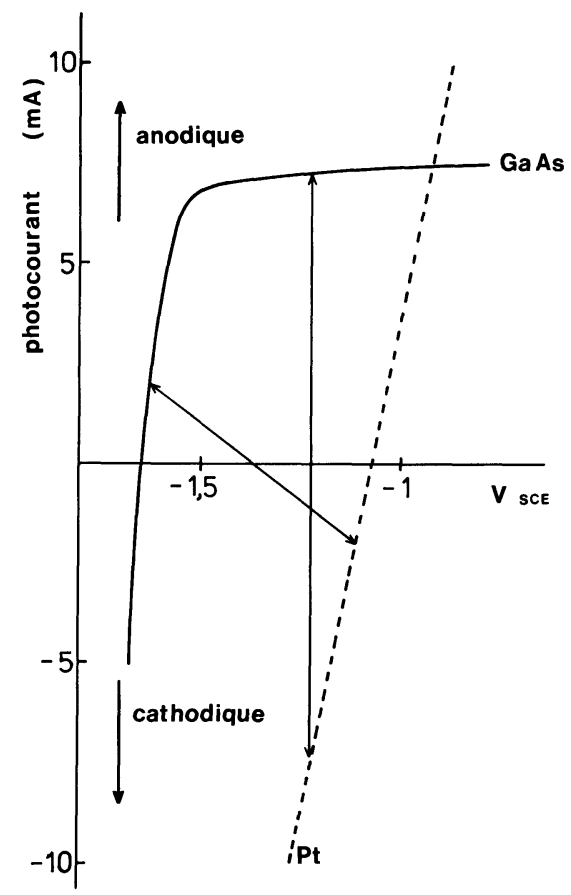

Fig. 10. - Caractéristique intensité-potentiel mesurée sous éclairement sur une électrode en GaAs-n dans un électrolyte contenant le couple redox $\mathrm{Se}^{2-} / \mathrm{Se}_{n}^{2-}$. La caractéristique de la contre-électrode est également reportée sur la figure. La cellule complète fonctionne aux extrémités des droites de charge indiquées par les flèches; flèches verticales : fonctionnement en court-circuit, flèches obliques : fonctionnement avec une résistance de charge.

[Current voltage characteristic measured under illumination on a n-type GaAs electrode in $\mathrm{Se}^{2-} / \mathrm{Se}_{n}^{2}$ containing electrolyte. The characteristic of the $\mathrm{Pt}$ counter-electrode has also been drawn on the figure. The whole cell operates at the extremities of the load lines at the arrows drawn on the figure. Vertical load line : short circuit; slanted load line : working with a load resistance, given by the slope of the line.] 
On peut donc déterminer, à l'aide de ces caractéristiques, la réponse courant-potentiel de la cellule complète. Celle-ci est reproduite sur la figure 11. Elle correspond à la cellule $\mathrm{GaAs} /\left(\mathrm{Se}^{2-} / \mathrm{Se}_{n}^{2-}\right) / \mathrm{Pt}$ sous un éclairement de $45 \mathrm{~mW} / \mathrm{cm}^{2}$ par la lampe à vapeur de Xe. Le rendement de conversion maximum est de $1,2 \%$. Une analyse chimique de l'électrolyte, après fonctionnement prolongé, a révélé la présence de gallium, ce qui montre que la stabilisation n'est pas parfaite.

Ceci est confirmé par la présence de piqûres de corrosion à la surface de l'électrode. La photodécomposition partielle de $\mathrm{GaAs}$ dans. $\mathrm{Se}^{2-} / \mathrm{Se}_{n}^{2-}$ a déjà été signalée par Ellis et al. [56].

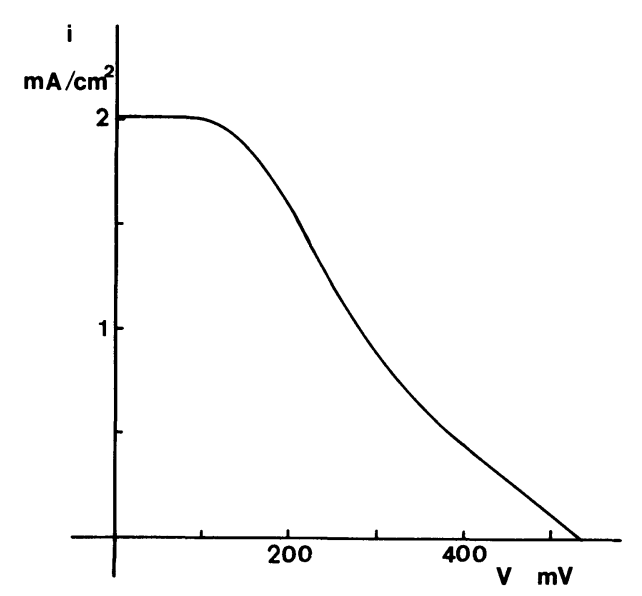

Fig. 11. - Réponse photocourant-phototension de la cellule $\mathrm{GaAs}-\mathrm{n} /\left(\mathrm{Se}^{2-} / \mathrm{Se}_{n}^{2-}\right) / \mathrm{Pt}$ sous éclairement de $45 \mathrm{~mW} / \mathrm{cm}^{2}$ par une lampe à vapeur de xénon.

[Output power characteristic of the $\mathrm{n}-\mathrm{GaAs} /\left(\mathrm{Se}^{2-} / \mathrm{Se}_{n}^{2-}\right) / \mathrm{Pt} \mathrm{PEC}$ cell under $45 \mathrm{~mW} / \mathrm{cm}^{2}$ illumination by a Xenon arc lamp.]

3.1.4 Importance de la préparation de la surface de l'électrode. - L'état de la surface de l'électrode est un facteur primordial pour la qualité du fonctionnement de la cellule PEC. Les mesures de capacité de l'interface semiconducteur-électrolyte ont montré l'importance de la préparation de la surface [71] : la grande dispersion des résultats obtenus à différentes valeurs de la fréquence à laquelle est mesurée la capacité peut être sensiblement réduite par une attaque chimique appropriée, qui a pour conséquence d'éliminer la couche désordonnée laissée à la surface par les traitements mécaniques préalables. La même attaque chimique améliore les caractéristiques intensité-potentiel et la réponse spectrale d'une cellule PEC [72]. Des mesures de réponse spectrale sous éclairement simultané d'une lampe à filament de tungstène filtrée par un monochromateur, et d'un laser destiné à pomper les électrons de la bande de valence, ont permis à Heller et al. [73, 74] de mettre en évidence l'existence de niveaux pièges situés dans la bande interdite près des bords de bande. Ces niveaux, qui ont été observés sur CdS, CdSe [73] et CdTe [74] disparaissent après une attaque chimique, et l'amélioration des performances de la cellule est très importante.

C'est par un traitement différent de la surface de l'électrode que Parkinson, Heller et Miller [58] sont parvenus à un rendement de conversion de $12 \%$ en éclairement solaire. Il a été obtenu en plongeant brièvement (environ $30 \mathrm{~s}$ ) l'électrode de GaAs dans une solution de ruthénium III. Les auteurs remarquent que l'attaque chimique, opérée avant ce traitement, joue sur le courant de court-circuit, alors que le traitement au $\mathrm{Ru}(\mathrm{III})$ accroît le photopotentiel en circuit ouvert. Parkinson et al. attribuent cette amélioration à un déplacement des états de surface situés près du bas de la bande de conduction [75]. Bolts et al. [76] ont eux obtenu une stabilisation de $\mathrm{Si}-\boldsymbol{n}$ en plongeant l'électrode pendant 1 à $3 \mathrm{~h}$ dans une solution de $\left(1,1^{\prime}\right.$ ferrocènediyl)-dichlorosilane.

Selon eux, il se forme au cours de ce traitement une monocouche liée chimiquement à la surface de l'électrode, et dans laquelle existe un couple redox : le couple ferrocène/ferrocénium, dont le niveau redox peut stabiliser le silicium. Le passage du courant se fait en deux étapes : échange électronique entre le silicium et la monocouche adsorbée, puis entre celle-ci et l'électrolyte. Ce type de configuration semble nécessaire pour la stabilisation du $\mathrm{Si}$ : on ne connaît pas en effet de couple redox soluble dans l'eau qui puisse le stabiliser (le couple ferrocène/ferrocénium n'est lui-même soluble que dans des liquides organiques). D'autre part, du fait de leur faible conductivité, il n'est pas envisageable d'utiliser un solvant organique pour une cellule solaire PEC. Avec la cellule au $\mathrm{Si} /($ ferrocène/ferrocénium), un rendement de $1,4 \%$ sous éclairement de $0,37 \mathrm{~mW} / \mathrm{cm}^{2}$ de la raie à $633 \mathrm{~nm}$ d'un laser $\mathrm{He}-\mathrm{Ne}$ a été mesuré. Ce rendement décroît lorsque l'éclairement augmente.

3.1.5 Cas particulier: les chalcogénures de métaux de transition. - En utilisant des chalcogénures de métaux de transition $\mathrm{MoS}_{2}$ [77] et $\mathrm{MoSe}_{2}$ [78, 79], Tributsch a obtenu des cellules PEC qui n'ont pas subi de dégradation après neuf mois de fonctionnement continu [80]. Il est apparu que contrairement à ce que laisserait présager la situation de leurs niveaux de décomposition [33], ces composés sont stables même en l'absence de couple redox stabilisateur. Leur utilisation en cellule de photoélectrolyse n'est cependant pas possible car ils y seraient attaqués par l'oxygène se dégageant à la photo-anode [81]. Cette stabilité est attribuée au fait que les états électroniques correspondant aux bords des bandes sont constitués par les orbitales d de l'atome de Mo, c'est-à-dire par des orbitales non liantes. Un échange électronique entre le semiconducteur et l'électrolyte ne met donc pas en jeu les électrons de liaison du cristal, et ne provoque pas sa décomposition. Un rendement de conversion de $3,5 \%$ a été obtenu avec une cellule $\operatorname{MoSe}_{2} /\left(\mathrm{I}_{2} / \mathrm{I}^{-}\right)$. La même stabilité a été 
d'autre part observée avec le séléniure de tungstène $\mathrm{WSe}_{2}$ [82].

3.2 Cellule de stockage. - La cellule de stockage se caractérise par l'emploi d'électrodes semiconductrices stables et par un électrolyte qui contient deux couples redox. L'énergie stockée correspond à la différence entre les niveaux redox de ces deux couples.

Le premier exemple en est la cellule de photoélectrolyse de Fujishima et Honda [1], utilisant une photoanode en $\mathrm{TiO}_{2}$ et l'eau comme électrolyte, avec les couples $\mathrm{O}_{2} / \mathrm{H}_{2} \mathrm{O}$ et $\mathrm{H}^{+} / \mathrm{H}_{2}$. Malheureusement, ainsi que l'ont montré Wrighton et al. [83], cette cellule ne peut fonctionner à l'énergie solaire seule. La décomposition de l'eau n'a lieu que lorsqu'on applique entre les électrodes une faible tension, de l'ordre de 0,3 V. Dans la cellule de Fujishima et Honda la photoélectrolyse était obtenue par l'emploi de deux électrolytes différents à l'anode et à la cathode : $\mathrm{H}_{2} \mathrm{SO}_{4} 1 \mathrm{M}$ à la première et $\mathrm{NaOH} 1 \mathrm{M}$ à la seconde, ce qui revient à remplacer la différence de potentiel par une différence de $\mathrm{pH}$, qui tend à se réduire au cours du fonctionnement de la cellule, avec arrêt de la photoélectrolyse.

Un schéma énergétique de la cellule de photoélectrolyse est donné par la figure 12 . Une condition nécessaire à son fonctionnement est que le niveau $\mathrm{du}$ bas de la bande de conduction à la surface du semiconducteur, $E_{\mathrm{c}}^{\mathrm{s}}$, soit supérieur au niveau $\mathrm{H}^{+} / \mathrm{H}_{2}$. En se reportant à la figure 5 , on voit que seuls $\mathrm{SrTiO}_{3}$ et $\mathrm{KTaO}_{3}$ seront capables de provoquer la photoélectrolyse pure $(\mathrm{CdS}, \mathrm{InP}, \mathrm{GaP}$ et $\mathrm{GaAs}$ sont à exclure car ils sont instables). Avec les autres semiconducteurs il sera nécessaire d'utiliser une tension

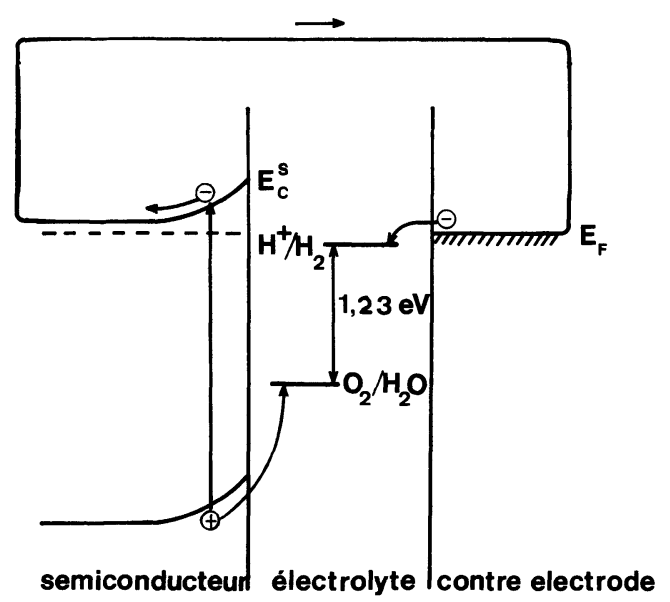

Fig. 12. - Schéma énergétique d'une cellule de photoélectrolyse de l'eau avec anode semiconductrice de type $n$ et contre-électrode métallique. Cette cellule ne peut fonctionner que si le niveau du bas de la bande de conduction à la surface du semiconducteur $E_{\mathrm{c}}^{\mathrm{s}}$, est supérieur au niveau $\mathrm{H}^{+} / \mathrm{H}_{2}$.

[Energy diagram of a photoelectrolysis cell with a semiconductor anode and a metal counter-electrode. This cell cannot work unless the conduction band edge $E_{\mathrm{c}}^{\mathrm{s}}$ at the semiconductor surface is greater than the $\mathrm{H}^{+} / \mathrm{H}_{2}$ redox level.] extérieure, d'autant plus grande que la différence entre $E_{\mathrm{c}}^{\mathrm{s}}$ et le niveau $\mathrm{H}^{+} / \mathrm{H}_{2}$ est importante.

3.2.1 Semiconducteurs stables. - Le tableau II donne la liste des semiconducteurs stables, en polarisation anodique pour le type $\mathrm{n}$ et cathodique pour le type $\mathrm{p}$, qui ont fait l'objet d'étude en cellule PEC. Pour $\mathrm{TiO}_{2}$ on pourra trouver des références plus complètes dans [35] et [84].

Tableau II. - Semiconducteurs utilisés dans les cellules de stockage.

\begin{tabular}{|c|c|c|c|c|}
\hline \multirow{3}{*}{$\begin{array}{c}\text { Semi- } \\
\text { conducteur }\end{array}$} & \multirow{2}{*}{$\begin{array}{c}\text { Bande } \\
\text { interdite } \\
\mathrm{eV}\end{array}$} & \multicolumn{2}{|c|}{$\begin{array}{l}\text { Potentiel de } \\
\text { bande plate }\end{array}$} & \multirow{3}{*}{ Références } \\
\hline & & $V_{\text {sce }}$ & $\mathrm{pH}$ & \\
\hline & - & - & - & \\
\hline \multicolumn{5}{|l|}{ Type $n$} \\
\hline $\mathrm{ZrO}_{2}$ & 5,0 & $-1,5$ & 7 & [88] \\
\hline $\mathrm{Ta}_{2} \mathrm{O}_{5}$ & 4,0 & $-1,1$ & 7 & [88] \\
\hline $\mathrm{SnO}_{2}$ & 3,5 & $-0,7$ & 13 & {$[18,89]$} \\
\hline $\mathrm{KTaO}_{3}$ & 3,5 & $-1,3$ & 13 & {$[18,87]$} \\
\hline $\mathrm{Nb}_{2} \mathrm{O}_{5}$ & 3,4 & $-0,8$ & 7 & [88] \\
\hline $\mathrm{SrTiO}_{3}$ & 3,4 & $-1,4$ & 13 & {$[18,85,86,90,91]$} \\
\hline $\mathrm{BaTiO}_{3}$ & 3,3 & $-0,45$ & 12,2 & {$[19,91-93]$} \\
\hline $\mathrm{TiO}_{2}$ & 3,0 & $-0,7$ & 13 & {$[1,18,35,91,94,117]$} \\
\hline $\mathrm{FeTiO}_{3}$ & 2,9 & $+0,1$ & 14 & {$[95,96]$} \\
\hline $\mathrm{WO}_{3}\left({ }^{1}\right)$ & 2,7 & $+0,2$ & 7 & {$[22,91,94,97-101]$} \\
\hline $\mathrm{YFeO}_{3}$ & 2,6 & $-0,5$ & 14 & [102] \\
\hline $\mathrm{Fe}_{2} \mathrm{O}_{3}\left({ }^{2}\right)$ & 2,2 & $-0,4$ & 8,6 & {$[20,91,94,103-108]$} \\
\hline \multicolumn{5}{|l|}{ Type $p$} \\
\hline $\mathrm{SiC}$ & 3,0 & $+1,1$ & 14 & [109] \\
\hline $\mathrm{GaP}$ & 2,3 & $+0,2$ & 9 & {$[109,111-114,116]$} \\
\hline $\mathrm{CdTe}$ & 1,4 & 0,0 & 14 & {$[109,110,114,115]$} \\
\hline GaAs & 1,4 & $-0,2$ & 9,2 & [109] \\
\hline InP & 1,3 & $+0,5$ & 9,2 & [109] \\
\hline
\end{tabular}

( $\left.{ }^{1}\right)$ Stable à $\mathrm{pH}<7$.

(2) Stable à $\mathrm{pH}>4,5$.

La référence [84] donne d'autre part une description détaillée des modes de fabrication des différents oxydes semiconducteurs. Outre les monocristaux, des polycristaux obtenus de diverses façons ont été utilisés :

- Oxydation thermique d'une feuille métallique $[94,97,100,101,107,108]$.

- Frittage $[93,106]$.

- Oxydation anodique [88, 108].

- Dépôt chimique en phase vapeur (C.V.D.) $[94,103]$.

- Pulvérisation cathodique [99].

- Dépôt d'un sel sur un substrat, suivi d'un chauffage sur une flamme [94].

Les performances de ces polycristaux sont en général comparables à celles obtenues avec des monocristaux [117].

On remarque que tous les semiconducteurs de type $\mathrm{n}$ stables sont des oxydes de métaux de transition. Leur grande largeur de bande interdite, supérieure à $3 \mathrm{eV}$ en général, les rend peu aptes à la conversion de l'énergie solaire. Seuls sont intéressants de ce point de vue $\mathrm{WO}_{3}(2,7 \mathrm{eV})$ et $\mathrm{Fe}_{2} \mathrm{O}_{3}(2,2 \mathrm{eV})$, ce 
dernier ayant de plus l'avantage d'un potentiel de bande plate plus cathodique.

3.2.2 Semiconducteurs protégés. - On a essayé de protéger de la photodécomposition des semiconducteurs de type $\mathbf{n}$ à bande interdite moins large en les recouvrant soit avec un métal [118] soit avec un semiconducteur stable $\left(\mathrm{TiO}_{2}\right.$ en général) [119-122]. Il semble toutefois que la protection ne soit efficace que lorsque la couche protectrice est trop épaisse pour permettre les échanges électroniques entre le semiconducteur protégé et l'électrolyte [121]. Ainsi, lorsqu'on observe un photocourant avec une électrode protégée par un dépôt métallique (or ou platine), celui-ci serait dû à la photodécomposition du semiconducteur, rendue possible par la porosité de la couche de protection, et non à l'oxydation de l'électrolyte [123].

De même, lorsque la protection par $\mathrm{TiO}_{2}$ est efficace, le photocourant mesuré correspond à celui d'une électrode en dioxyde de titane et non à celui du semiconducteur protégé [122].

3.2.3 Cellule composée de deux photoélectrodes semiconductrices. - L'efficacité de la cellule de stockage est améliorée par l'emploi de deux électrodes semiconductrices au lieu d'une : une photoanode de type $n$ et une photocathode de type $p$ [111-114]. La figure 13 montre le schéma énergétique obtenu. On

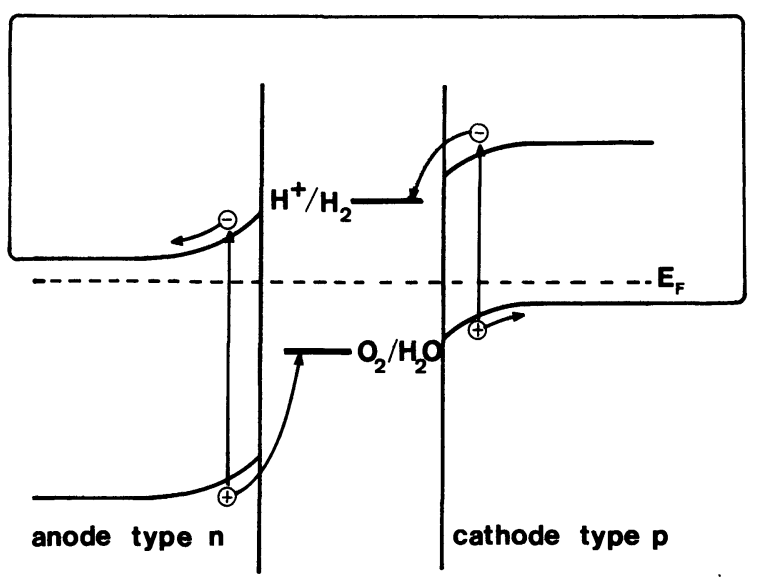

Fig. 13. - Schéma énergétique d'une cellule de photoélectrolyse à deux électrodes semiconductrices : une anode de type $\mathrm{n}$ et une cathode de type $\mathrm{p}$.

[Energy diagram of a double semiconductor cell : n-type anode and p-type cathode.]

voit que bien que le niveau $E_{\mathrm{c}}^{\mathrm{s}}(\mathrm{n})$ de la photoanode soit inférieur (plus anodique) au niveau $\mathrm{H}^{+} / \mathrm{H}_{2}$, la photoélectrolyse sera possible grâce à l'apport énergétique des photoélectrons arrivant à la surface de la photocathode. Ceci a été effectivement observé sur la cellule $\mathrm{TiO}_{2}$-n/GaP-p [111-114] qui permet la photoélectrolyse de l'eau sans polarisation extérieure. Les caractéristiques intensité-potentiels des deux électrodes de cette cellule sont données par la figure 14

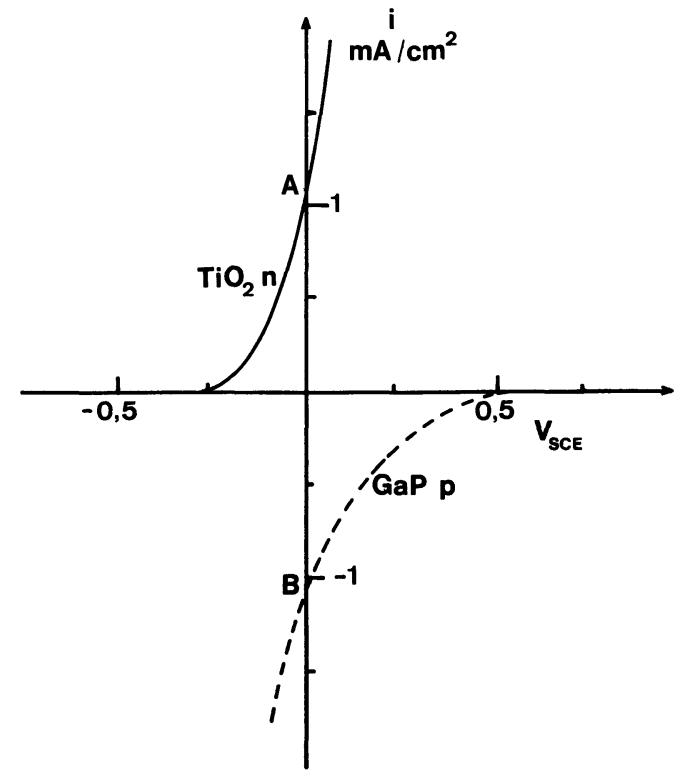

Fig. 14. - Caractéristique intensité-potentiel sous éclairement des deux électrodes d'une cellule de photoélectrolyse $\mathrm{TiO}_{2}-\mathrm{n} / \mathrm{GaP}-\mathrm{p}$. La cellule complète fonctionne en court-circuit entre les points A et $\mathbf{B}$.

[Current-voltage characteristics measured under illumination of each electrode of a $\mathrm{n}-\mathrm{TiO}_{2} / \mathrm{p}-\mathrm{GaP}$ photoelectrolysis cell. The whole cell works in short-circuit between points $A$ and $B$.]

[113]. A la différence de la cellule autorégénérée, la cellule de stockage donne son meilleur rendement en court-circuit, c'est-à-dire entre les points $\mathrm{A}$ et $\mathrm{B}$ de la figure 14.

Le rendement de conversion $\eta$ d'une cellule de stockage est plus difficile à définir que celui d'une cellule photovoltaïque. La définition suivante est le plus souvent adoptée

$$
\eta=i\left(V_{\mathrm{st}}-V_{\mathrm{ext}}\right) / W_{\mathrm{ph}}
$$

où $i$ est la densité de photocourant, $V_{\text {st }}$ la différence entre les niveaux redox, $V_{\text {ext }}$ le potentiel extérieur (lorsque celui-ci est nécessaire) et $W_{\text {ph }}$ l'énergie lumineuse reçue par unité de surface par la (ou les) photoélectrode(s). $V_{\text {st }}$ et $V_{\text {ext }}$ étant des données de la cellule, dépendant de la nature de l'électrolyte et des photoélectrodes, on voit que le rendement maximum est obtenu lorsque $i$ est maximum, c'est-à-dire en court-circuit. Pour la cellule de photoélectrolyse,

$$
V_{\mathrm{st}}=V_{\mathrm{H}_{2}}=1,23 \mathrm{~V} \text {. }
$$

Les rendements des cellules de stockage sont très faibles en général. Ohashi, McCann et Brockris [114] ont mesuré le rendement de conversion de trois cellules à double photoélectrode éclairées par une lampe à vapeur de Xe. La plus forte valeur a été obtenue par la cellule $\mathrm{SrTiO}_{3}-\mathrm{n} / \mathrm{GaP}-\mathrm{p}: 0,67 \%$. Au soleil cette valeur tombe à $0,001 \%$ du fait de la très faible absorption de $\mathrm{SrTiO}_{3}$. Nozik [112] a estimé le rendement solaire de la cellule $\mathrm{TiO}_{2}$-n/GaP-p à $0,25 \%$. 
3.2.4 Autres types de cellule de stockage. - Les cellules de stockage autres que les cellules de photoélectrolyse ont été très peu étudiées. Halmann [124] a décrit une cellule réalisant la réduction de $\mathrm{CO}_{2}$ à la surface d'une photocathode en GaP-p. Les produits obtenus sont l'acide formique, le formaldéhyde et le méthanol. Une tension extérieure de $-0,8$ à $-0,9 \mathrm{~V}$ doit être appliquée entre les électrodes. Le rendement de conversion, défini par la relation (9), est de 0,97\% pour la formation du formaldéhyde et $0,61 \%$ pour celle du méthanol.

Signalons pour terminer une cellule de stockage, réalisée par Manassen, Hodes et Cahen [125], qui se rapproche plus d'une cellule autorégénérée que de celles que nous venons de décrire. Elle comprend une photoanode en CdSe polycristallin stabilisée par des sulfures, et deux contre-électrodes.

La première est en carbone et baigne dans le même électrolyte que la photoanode, tandis que la seconde, en étain, en est séparée par une membrane à perméabilité sélective. Dans le noir l'anode semiconductrice est connectée à l'électrode d'étain et l'ensemble fournit du courant électrique avec consommation de l'électrode d'étain. Sous illumination, la photoanode est reliée à la contre-électrode en carbone et l'on observe une régénération de l'électrode d'étain. On a ainsi réalisé une batterie solaire capable de se recharger par simple exposition au rayonnement solaire.

4. Conclusion. - Nos conclusions ne seront pas les mêmes pour les deux types de cellule solaire PEC.

Les cellules autorégénérées concurrencent directement les cellules photovoltaïques. Leur avantage tient en la simplicité de leur mise en œuvre, puisqu'elles ne nécessitent pas d'élaboration utilisant les technologies délicates et coûteuses que demandent les jonctions solide-solide : la jonction est obtenue par simple contact avec l'électrolyte. Un rendement de $12 \%$ a été atteint avec une électrode en GaAs monocristallin [58]. Les cellules PEC autorégénérées deviendront intéressantes lorsqu'un rendement comparable pourra être obtenu avec des électrodes en couche mince, polycristalline ou amorphe. Leur prix de revient pourrait les rendre alors plus avantageuses que les cellules photovoltaïques. Une amélioration $\mathrm{du}$ rendement énergétique de ce type de cellule pourrait être obtenu par des traitements appropriés de la surface du semiconducteur.

Le très faible rendement actuel des cellules de stockage fait qu'elles sont, pour l'instant, moins intéressantes que, par exemple, une cellule d'électrolyse alimentée par des photopiles solaires. La nouveauté des phénomènes liés à leur fonctionnement et des matériaux semiconducteurs utilisés, fait que les progrès sont plus lents que ceux de la cellule autorégénérée. Il nous semble trop tôt pour prédire leur avenir.

Il existe un troisième type de cellule PEC dont nous n'avons pas parlé : les cellules de photoélectrocatalyse et photoélectrosynthèse [3].

Dans ces cellules, l'électrode semiconductrice n'est pas reliée à une contre-électrode. Il y a à la fois réaction d'oxydation (par les trous) et de réduction (par les électrons) à sa surface. Le semiconducteur peut alors être utilisé sous forme de poudre dispersée au sein de l'électrolyte. Ce type de cellule peut être utilisé pour le traitement de déchets et la dépollution [126], ou par la production de combustibles stockables (méthane à partir de l'acide acétique [127], éthane à partir d'acétate [128]). En s'inspirant de ce type de cellule, on peut imaginer des cellules de stockage d'un genre nouveau.

On pourrait, par exemple, platiner partiellement les particules d'un semiconducteur en poudre, de façon à obtenir des microcellules PEC [3]. L'absorption optique d'un tel système pourrait être élevée, et le fait qu'il fonctionne en court-circuit ne peut qu'améliorer son rendement.

Un dispositif comparable a été imaginé par Nozik [129] : la diode photochimique, constituée de deux semiconducteurs de type $n$ et $p$, accolés par leur face interne par l'intermédiaire d'un contact ohmique. En dispersant un grand nombre de ces diodes au sein d'un électrolyte, on obtient une cellule de photoélectrolyse particulièrement simple, dont le seul problème serait de séparer les deux gaz obtenus. L'avenir des cellules de stockage est peut-être dans ce genre de dispositif, fort différent on le voit de la cellule photovoltaïque.

\section{Notations}

$C_{\mathrm{H}} \quad$ : Capacité de la couche de Helmholtz (par unité de surface).

$C_{\text {sc }} \quad$ : Capacité de la zone de charge d'espace (par unité de surface).

$e \quad$ : Charge de l'électron.

$E_{\mathrm{c}} \quad$ : Niveau du bas de la bande de conduction.

$E_{\mathrm{v}} \quad$ : Niveau du haut de la bande de valence.

$E_{\mathrm{F} / \mathrm{sc}}$ : Niveau de Fermi du semiconducteur.

$E_{\mathrm{F} / \text { redox }}$ : Niveau redox dans l'électrolyte.

$E_{\mathrm{g}} \quad$ : Largeur de la bande interdite.

$i, j \quad$ : Densité de courant.

$k \quad$ : Constante de Boltzmann.

$L_{\mathrm{p}} \quad$ : Longueur de diffusion des porteurs minoritaires.

$N \quad$ : Densité des porteurs majoritaires (par unité de volume).

NHE : Electrode normale à hydrogène.

$p \quad$ : Densité des porteurs minoritaires (par unité de volume).

SCE : Electrode au calomel saturé.

$T \quad$ : Température absolue.

$V \quad$ : Potentiel d'électrode (par rapport à une électrode de référence).

$V_{\mathrm{fb}} \quad$ : Potentiel de bandes plates.

$W \quad$ : Largeur de la zone de charge d'espace. 
$\alpha \quad$ : Coefficient d'absorption optique.

$\Delta V_{\mathbf{H}} \quad$ : Chute de potentiel dans la couche de Helmholtz.

$\Delta V_{\text {sc }} \quad$ : Chute de potentiel dans la zone de charge $\varphi_{0}$ d'espace.
$\Delta V_{\mathrm{ph}} \quad:$ Photopotentiel.

$\varepsilon \quad:$ Constante diélectrique.

$\varepsilon_{0} \quad:$ Permittivité du vide.

$\varphi_{0} \quad$ : Flux de photons incidents (par unité de surface et par unité de temps).

\section{Bibliographie}

[1] Fujishima, A. et Honda, K., Nature 238 (1972) 37.

[2] Gerischer, H., J. Electroanal. Chem. 58 (1975) 263.

[3] BARD, A. J., 2nd International Conference on the Photochemical Conversion and storage of Solar Energy, Cambridge, 10-12 août 1978, Photochem. 10 (1979) 59.

[4] Photovoltaic Solar Energy Conference, Proceedings of the International conference held at Luxembourg, 27-30 septembre 1977 (D. Reider Publishing Company, Dordrecht, Pays-Bas) 1977.

[5] Semiconductor Liquid Junction Solar Cells, Proceedings of a Conference held at Airlie, Virgina, 3-5 mai 1977, Heller, A., Ed. (The Electrochemical Society, Princeton, New Jersey) 1977.

[6] Clechet, P., Martelet, C., Martin, J.-R. et Olier, R., L'actualité chimique, novembre (1978) p. 17.

[7] Gerischer, H. dans Physical Chemistry, Vol. IX A, Eyring, H., Henderson, D. et Jost, W., Ed. (Academic Press, New York) 1970, p. 463.

[8] LohmanN, F., Z. Naturforsch. 22a (1967) 813.

[9] Gomer, R. et Tryson, G., J. Chem. Phys. 66 (1977) 4413.

[10] Myamlin, V. A. et Pleskov, Yu. V., Electrochemistry of Semiconductors (Plenum, New York) 1967, p. 30.

[11] Lohmann, F., Ber. Bunsenges. Phys. Chem. 70 (1966) 428.

[12] Memming, R., Philips Res. Repts. 19 (1964) 323.

[13] Dewald, F., Bell Syst. Techn. J. 39 (1960) 615.

[14] Horowitz, G., J. Appl. Phys. 49 (1978) 3571.

[15] Ginley, D. S. et Butler, M. A., J. Electrochem. Soc. 125 (1978) 1968

[16] Sze, S. M., Physics of Semiconductor Devices (John Wiley and Sons, New York) 1969, p. 25.

[17] Cardon, F. et Gomes, W. P., J. Phys. D 11 (1978) L 63.

[18] Bolts, J. M. et Wrighton, M. S., J. Phys. Chem. 80 (1976) 2641.

[19] Kennedy, J. H. et Freze, F. W. Jr., J. Electrochem. Soc. 123 (1976) 1683.

[20] Quinn, R. K. et NASBy, R. D., Mat. Res. Bull. 11 (1976) 1011.

[21] Butler, M. A. et Ginley, D. S., J. Electrochem. Soc. 125 (1978) 228.

[22] Butler, M. A., J. Appl. Phys. 48 (1977) 1914.

[23] Lemasson, P. et Gautron, J., C. R. Hebd. Séan. Acad. Sci., Série C, 288 (1979) 149.

[24] Gurney, R. W., Proc. Roy. Soc., A 134 (1931) 137.

[25] Gerischer, H., Surf. Sci. 18 (1969) 97.

[26] Van den Berghe, R. A. L., Cardon, F. et Gomes, W. P., Surf. Sci. 39 (1973) 368.

[27] Gärtner, W. W., Phys. Rev. 116 (1959) 84.

[28] Wilson, R. H., J. Appl. Phys. 48 (1977) 4292.

[29] LASÉR, D. et BARD, A. J., J. Electrochem. Soc. 123 (1976) 1837.

[30] Reiss, H., J. Electrochem. Soc. 125 (1978) 937.

[31] Gerischer, H., J. Electroanal. Chem. 82 (1977) 133.

[32] BARD, A. J. et Wrighton, M. S., J. Electrochem. Soc. 124 (1977) 1706.

[33] Gerischer, H., J. Vac. Sci. Technol. 15 (1978) 1422.

[34] Williams, F. et NozIK, A. J., Nature 271 (1978) 137.

[35] NozIK, A. J., Ann. Rev. Phys. Chem. 29 (1978) 189.

[36] Williams, F., Varma, S. et Hillenius, S., J. Chem. Phys. 64 (1976) 1549.

[37] Memming, R., J. Electrochem. Soc. 125 (1978) 117.
[38] Ellis, A. B., Kaiser, S. W. et Wrighton, M. S., J. Am. Chem. Soc. 98 (1976) 1635.

[39] Ellis, A. B., Kaiser, S. W. et Wrighton, M. S., J. Am Chem. Soc. 98 (1978) 6855.

[40] Heller, A., Chang, K. C. et Miller, B., J. Electrochem. Soc. 124 (1977) 697.

[41] Minoura, H., Tsuiki, M. et OKi, T., Ber. Bunsenges. Phys. Chem. 81 (1977) 588.

[42] Miller, B. et HelleR, A., Nature 262 (1976) 680.

[43] Peter, L. M., Electrochim. Acta 23 (1978) 1073.

[44] Tsuiki, M., Minoura, H., Nakamura, T. et Ueno, Y., J. Appl. Electrochem. 8 (1978) 523.

[45] Chartier, P., Fall, S. S., Faye, M., Cadene, M. et CohenSolal, G. W., C. R. Hebd. Séan. Acad. Sci., Série C, 284 (1977) 437.

[46] Tsuiki, M. et Minoura, H., Chem. Lett. (1978) 113.

[47] Inoue, T., Watanabe, T., Fujishima, A. et Honda, K., J. Electrochem. Soc. 124 (1977) 719.

[48] Ellis, A. B., Kaiser, S. W., Bolts, J. M. et Wrighton, M. S., J. Am. Chem. Soc. 99 (1977) 2839.

[49] Tsuiki, M., Ueno, Y., Nakamura, T. et Minoura, H., Chem. Lett. (1978) 289.

[50] Noufi, R. N., KoHL, P. A. et BARD, A. J., J. Electrochem. Soc. 125 (1978) 375.

[51] Miller, B., Heller, A., Robbins, M., Menezes, S., Chang, K. C. et Thomson, J. Jr., J. Electrochem. Soc. 124 (1977) 1019.

[52] Gerrard, W. A. et Owen, J. R., Mat. Res. Bull. 12 (1977) 677.

[53] Heller, A., Chang, K. C. et Miller, B., J. Am. Chem. Soc. 100 (1978) 684.

[54] Ellis, A. B., Kaiser, S. W. et Wrighton, M. S., J. Am. Chem. Soc. 98 (1976) 6418

[55] Danaher, W. J. et Lyons, L. E., Nature 271 (1978) 139.

[56] Ellis, A. B., Bolts, J. M., Kaiser, S. W. et Wrighton, M. S., J. Am. Chem. Soc. 99 (1976) 2848.

[57] Chang, K. C., Heller, A., Schwartz, B., Menezes, S. et Miller, B., Science 196 (1977) 1097.

[58] Parkinson, B. A., Heller, A. et Miller, B., Appl. Phys. Lett. 33 (1978) 521.

[59] Ellis, A. B., Bolts, J. M. et Wrighton, M. S., J. Electrochem. Soc. 124 (1977) 1603.

[60] Robbins, M., Bachmann, K. J., Lambrecht, V. G., Thiel, F. A., Thomson, J. Jr., Vadimsky, R. G., Menezes, S., Heller, A. et Miller, B., J. Electrochem. Soc. 125 (1978) 831.

[61] Hill, J. E. et Chamberlin, R. R., Process for Making Conductive Films, U.S. Patent no 3 148.084, 8 septembre 1964.

[62] Minoura, H., Nakamura, T., Ueno, Y. et Tsuiki, M., Chem. Lett. (1977) 913.

[63] Chartier, P., Fall, S. S., Faye, M., Cadene, M. et CohenSolal, G. W., Réf. [3] (1977) p. 1192.

[64] Wrighton, M. S., Bolts, J. M., Bocarsly, A. B., Palazzotto, M. C. et Walton, E. G., J. Vac. Sci. Technol. 15 (1978) 1429.

[65] Bockris, J. O. M. et Reddy, A. K. N., Modern Electrochemistry (Plenum, New York) 1970, p. 1070.

[66] Gerischer, H. et Gobrecht, J., Ber. Bunsenges. Phys. Chem. 82 (1978) 520. 
[67] Heller, A., Schwartz, C. P., Vadimsky, R. G., Menezes, S. et Miller, B., J. Electrochem. Soc. 125 (1978) 1157.

[68] Cahen, D., Hodes, G., et Manassen, J., J. Electrochem. Soc. 125 (1978) 1623.

[69] Stirn, R. J. et YeH, Y. C. M., Appl. Phys. Lett. 27 (1975) 95.

[70] Noufi, R. N., KoHl, P. A., Rogers, J. W. Jr., White, J. M. et BARD, A. J., J. Electrochem. Soc. 126 (1979) 949.

[71] Laflere, W. H., Van Meirhaeghe, R. L., Cardon, F. et Gomes, W. P., Surf. Sci. 59 (1976) 401.

[72] Van Meirhaeghe, R. L., Cardon, F. et Gomes, W. P., Ber. Bunsenges. Phys. Chem. 83 (1979) 239.

[73] Heller, A., Chang, K. C. et Miller, B., J. Electrochem. Soc. 124 (1977) 697.

[74] Heller, A., Chang, K. C. et Miller, B., J. Am. Chem. Soc. 100 (1978) 684.

[75] Parkinson, K. A., Heller, A. et Miller, B., J. Electrochem. Soc. 126 (1979) 954.

[76] Bolts, J. M., Bocarsly, A. B., Palazzotto, M. C., Walton, E. G., Lewis, N. S. et Wrighton, M. S., J. Am. Chem. Soc. 101 (1979) 1378.

[77] Tributsch, H., Ber. Bunsenges. Phys. Chem. 81 (1977) 361.

[78] Tributsch, H., Ber. Bunsenges. Phys. Chem. 82 (1978) 169.

[79] Tributsch, H., J. Electrochem. Soc. 125 (1978) 1086.

[80] Gobrecht, J., Tributsch, H. et Gerischer, H., J. Electrochem. Soc. 125 (1978) 2085.

[81] Tributsch, H., Z. Naturforsch. 32a (1977) 972.

[82] Gobrecht, J., Gerischer, H. et TributsCh, H., Ber. Bunsenges. Phys. Chem. 82 (1978) 1331.

[83] Wrighton, M. S., Ginley, D. S., Wolczanski, P. T., Ellis, A. B., Morse, D. L. et Linz, A., Proc. Nat. Acad. Sci. U.S.A. 72 (1975) 1518.

[84] Tomkiewicz, M. et FAy, H., Appl. Phys. 18 (1979) 1.

[85] Wrighton, M. S., Ellis, A. B., Wolczanski, P. T., Morse, D. L., Abrahamson, M. B. et Ginley, D. S., J. Am. Chem. Soc. 98 (1976) 2774.

[86] Mavroides, J. G., Kafalas, J. A. et Kolesar, D. F., Appl. Phys. Lett. 28 (1976) 241.

[87] Ellis, A. B., Kaiser, S. W. et Wrighton, M. S., J. Phys. Chem. 80 (1976) 1325.

[88] Clechet, P., Martin, J. R., Olier, R. et Vallouy, C., C. R. Hebd. Séan. Acad. Sci., Série C, 282 (1976) 887.

[89] Wrighton, M. S., Morse, D. L., Ellis, A. B., Ginley, D. S. et Abrahamson, H. B., J. Am. Chem. Soc. 98 (1976) 44.

[90] Watanabe, T., Fujishima, A. et Honda, K., Bull. Chem. Soc. Jap. 49 (1976) 355.

[91] Kung, H. H., Jarett, H. S., Sleight, A. W. et Ferretti, A., J. Appl. Phys. 48 (1977) 2463.

[92] Nasby, R. D., Quinn, R. K., Mat. Res. Bull. 11 (1976) 985.

[93] Kennedy, J. H. et Freze, K. W. Jr., J. Electrochem. Soc. 123 (1976) 1683.

[94] Hardee, K. L. et Bard, A. J., J. Electrochem. Soc. 124 (1977) 215.

[95] Ginley, D. S. et Butler, M. A., J. Appl. Phys. 48 (1977) 2019.

[96] Morosin, B., Baughman, R. J., Ginley, D. S. et Butler, M. A., J. Appl. Cryst. 11 (1978) 121.

[97] Hodes, G., CAHEN, D. et MANASSEN, J., Nature 260 (1976) 312 .
[98] Butler, M. A., NASby, R. D. et Quinn, R. K., Solid State Commun. 19 (1976) 1011.

[99] Gissler, W. et Memming, R., J. Electrochem. Soc. 124 (1977) 1710.

[100] Derrington, C. E., Godek, W. S., Castro, C. A. et Wold, A., Inorg. Chem. 17 (1978) 977.

[101] Gerrard, W. A., J. Electroanal. Chem. 86 (1978) 421.

[102] Butler, M. A., Ginley, D. S. et Eibschutz, M., J. Appl. Phys. 48 (1977) 3070.

[103] Hardee, K. L. et BARD, A. J., J. Electrochem. Soc. 123 (1976) 1024.

[104] Yeh, L. R. et Hackerman, N., J. Electrochem. Soc. 124 (1977) 833.

[105] Kennedy, J. H. et Freze, K. W. Jr., J. Electrochem. Soc. 125 (1978) 709.

[106] McGregor, K. G., Calvin, M. et Otvos, J. W., J. Appl. Phys. 50 (1979) 369.

[107] Curran, J. S. et Gissler, W., J. Electrochem. Soc. 126 (1979) 56.

[108] Wilhem, S. M., Yun, K. S., Ballenger, L. W. et HaCkeRMAN, N., J. Electrochem. Soc. 126 (1979) 419.

[109] Bockris, J. O'M. et Uosaki, K., J. Electrochem. Soc. 124 (1977) 1348.

[110] Ohashi, K., Uosaki, K. et Bockris, J. O’M., Int. J. Energy Res. 1 (1977) 25.

[111] Yoneyama, H., Sakamoto, H. et Tamura, H., Electrochim. Acta 20 (1975) 341.

[112] NozIK, A. J., Appl. Phys. Lett. 29 (1976) 150.

[113] Bourrasse, A. et Horowitz, G., J. Physique-Lett. 38 (1977) L-291.

[114] Ohashi, K., McCann, J. et Bockris, J. O'M., Int. J. Energy Res. 1 (1977) 259.

[115] Bolts, J. M., Ellis, A. B., LegG, K. D. et Wrighton, M. S. J. Am. Chem. Soc. 99 (1977) 4826.

[116] Tomkiewicz, M. et Woodall, J. M., Science 196 (1977) 990.

[117] Dutoit, E. C., Cardon, F., Van den Kerchove, F. et Gomes, W. P., J. Appl. Electrochem. 8 (1978) 247.

[118] Nakato, Y., AвE, K. et Tsubomura, H., Ber. Bunsenges. Phys. Chem. 80 (1976) 1002.

[119] Bockris, J. O’M. et UosaKi, K., Energy 1 (1976) 95.

[120] Morisaki, H., Watanabe, T., Iwase, M. et Yazawa, K., Appl. Phys. Lett. 29 (1976) 338.

[121] Kohl, P. A., Frank, S. N. et BARD, A. J., J. Electrochem. Soc. 124 (1977) 225.

[122] Tomkiewicz, M. et Woodall, J. M., J. Electrochem. Soc. 124 (1977) 1436.

[123] Harris, L. A., Gerstner, M. E. et Wilson, R. H., J. Electrochem. Soc. 124 (1977) 1511.

[124] Halmann, M., Nature 275 (1978) 115.

[125] Manassen, J., Hodes, G. et Cahen, D., J. Electrochem. Soc. 124 (1977) 532.

[126] Clechet, P., Martelet, C., Martin, J.-R. et Olier, P., C. R. Hebd. Séan. Acad. Sci., Série C, 287 (1978) 405.

[127] Kraeutler, B. et Bard, A. J., J. Am. Chem. Soc. 100 (1978) 2239.

[128] Kraeutler, B. et Bard, A. J., Nouv. J. Chim. 3 (1979) 31.

[129] NozIK, A. J., Appl. Phys. Lett. 30 (1977) 567. 